\title{
Translational control of the undifferentiated phenotype in ER-positive breast tumor cells: Cytoplasmic localization of ER $\alpha$ and impact of IRES inhibition
}

\author{
CHRISTOS VAKLAVAS ${ }^{1,2}$, KURT R. ZINN ${ }^{1-4}$, SHARON L. SAMUEL ${ }^{1,3}$, ZHENG MENG $^{1,5,6}$, \\ WILLIAM E. GRIZZLE ${ }^{1,4}$, HYOUNGSOO CHOI ${ }^{1,2,7}$ and SCOTT W. BLUME ${ }^{1,2,5}$ \\ ${ }^{1}$ Comprehensive Cancer Center and Departments of ${ }^{2}$ Medicine, Division of Hematology and Oncology, \\ ${ }^{3}$ Radiology, ${ }^{4}$ Pathology and ${ }^{5}$ Biochemistry and Molecular Genetics, University of Alabama at Birmingham, \\ Birmingham, AL 35294, USA
}

Received October 10, 2017; Accepted February 12, 2018

DOI: 10.3892/or.2018.6332

\begin{abstract}
Using a series of potential biomarkers relevant to mechanisms of protein synthesis, we observed that estrogen receptor (ER)-positive breast tumor cells exist in two distinct yet interconvertible phenotypic states (of roughly equal proportion) which differ in the degree of differentiation and use of IRES-mediated translation. Nascently translated IGF1R in the cytoplasm positively correlated with IRES activity and the undifferentiated phenotype, while epitope accessibility of RACK1, an integral component of the 40S ribosomal subunit, aligned with the more differentiated IRES-off state. When deprived of soluble growth factors, the entire tumor cell population shifted to the undifferentiated phenotype in which IRES-mediated translation was active, facilitating survival under these adverse microenvironmental conditions. However, if IRES-mediated translation was inhibited, the cells instead were forced to transition uniformly to the more differentiated state. Notably, cytoplasmic localization of estrogen receptor $\alpha$ $(\mathrm{ER} \alpha / \mathrm{ESR} 1)$ precisely mirrored the pattern observed with nascent IGF1R, correlating with the undifferentiated
\end{abstract}

Correspondence to: Dr Scott W. Blume, Comprehensive Cancer Center, University of Alabama at Birmingham, Bevill Biomedical Research Bldg, Room 765, 845 19th Street S, Birmingham, AL 35294, USA

E-mail: scblume704@gmail.com

Present addresses: ${ }^{6}$ Analytical Development Division, Five Prime Therapeutics, Inc., South San Francisco, CA 94080, USA; ${ }^{7}$ Department of Pediatrics, Seoul National University Bundang, Hospital Gyeonggi-do 463-707, Republic of Korea

Abbreviations: ER $\alpha$, estrogen receptor alpha; IGF1R, insulin-like growth factor 1 receptor; RACK1, receptor for activated C kinase 1; IRES, internal ribosome entry site

Key words: ER-positive breast cancer, estrogen receptor $\alpha, \mathrm{MYC}$, RACK1, IGF1R, connexin 43
IRES-active phenotype. Inhibition of IRES-mediated translation resulted in both a shift in ER $\alpha$ to the nucleus (consistent with differentiation) and a marked decrease in ER $\alpha$ abundance (consistent with the inhibition of ER $\alpha$ synthesis via its IRES). Although breast tumor cells tolerated forced differentiation without extensive loss of their viability, their reproductive capacity was severely compromised. In addition, CDK1 was decreased, connexin 43 eliminated and Myc translation altered as a consequence of IRES inhibition. Isolated or low-density ER-positive breast tumor cells were particularly vulnerable to IRES inhibition, losing the ability to generate viable cohesive colonies, or undergoing massive cell death. Collectively, these results provide further evidence for the integral relationship between IRES-mediated translation and the undifferentiated phenotype and demonstrate how therapeutic manipulation of this specialized mode of protein synthesis may be used to limit the phenotypic plasticity and incapacitate or eliminate these otherwise highly resilient breast tumor cells.

\section{Introduction}

Internal ribosome entry sites (IRESs) are translation-regulatory features found in association with the mRNAs encoding many cancer-relevant proteins (1-2). IRES-mediated translation is a specialized mode of protein synthesis which is independently regulated and remains operational even when the general protein synthesis mediated by ribosome scanning from the beginning of the mRNA is shut down (Fig. 1A). Malignant cells are particularly dependent on IRES-mediated translation and exploit this mechanism to synthesize oncogenic proteins to promote their own survival, particularly under adverse microenvironmental conditions such as those to which the tumor is exposed in vivo (3-7).

The inhibition of protein synthesis has been proven to be a highly effective therapeutic strategy. Many of our antibacterial antibiotics target the prokaryotic ribosome and interfere selectively with its function $(8,9)$. Anticancer agents targeting the mTOR function and thereby inhibiting conventional cap-dependent translation are showing considerable promise in ongoing clinical trials (10). Given its role in promoting 
tumor-cell survival under adverse conditions, there is reason to anticipate that selective inhibition of IRES-mediated translation could represent a highly effective anticancer strategy.

Our lab has been working on the development of a series of inhibitors of IRES-mediated translation, which could be used to probe the contribution of IRES-mediated translation to cancer pathogenesis and to assess the potential use of IRES inhibition as a therapeutic intervention. We recently described (11) the first of three IRES-inhibitor lead compounds (cpd_P), comparing and contrasting the effects on the IGF1R and MYC IRESs. We found that sustained IRES inhibition induced terminal differentiation in triple-negative breast cancer and other highly undifferentiated tumor types (12).

In the present study we examined the consequences of IRES inhibition on models of ER-positive human breast cancer, using the second of these lead compounds (cpd_W), which was found to be particularly effective against these cells. We observed that IRES inhibition forced these tumor cells to abandon the undifferentiated phenotype, resulting in terminal differentiation, incapacitation, or death of the malignant cells. Marked changes in ER $\alpha, \mathrm{CDK} 1$, connexin 43 and Myc were observed which correlated with these detrimental outcomes. In addition, potential biomarkers of the IRES-mediated translation were characterized which made it possible to directly visualize these phenotypic transitions. These findings have important implications for the biology of ER-positive breast cancer and reveal what may be accomplished by modulating IRES-mediated translation.

\section{Materials and methods}

Reagents and antibodies. IRES inhibitor lead compound W [cpd_W: Ethyl 2-\{(2-(1,3-benzoxazol-2-ylthio)butanoyl) amino\}-4-methyl-1,3-thiazole-5-carboxylate, MW 405] was originally identified from a high throughput screen of 135,000 compounds using T47D cells genetically engineered with a bicistronic construct containing the human IGF1R IRES (11). The compound was purchased from Chembridge Corporation (San Diego, CA, USA), subsequently resynthesized (97.9\% purity) and the new stock precisely recapitulated the biological activity of the original stock. Cycloheximide and puromycin were obtained from Sigma-Aldrich (St. Louis, MO, USA).

The primary antibodies used in these experiments are listed in Table I. The secondary antibodies for indirect immunofluorescence staining were AlexaFluor 488 or 594-conjugated goat anti-rabbit IgG, anti-mouse IgG, or anti-mouse IgM (for RACK1) (highly cross-adsorbed; Life Technologies; Thermo Fischer Scientific, Waltham, MA, USA). 4',6-Diamidino-2-phenylindole dihydrochloride (DAPI) was obtained from Sigma-Aldrich.

Cells, cell culture and treatment conditions. T47D cells were obtained from the American Type Culture Collection (ATCC, Manassas, VA, USA) and propagated using standard techniques in RPMI-1640 medium containing $10 \%$ fetal calf serum (FCS) and $10 \mu \mathrm{g} / \mathrm{ml}$ insulin. ZR-75-1 cells were a generous gift from Dr Patsy Oliver and propagated in RPMI-1640 with 20\% FCS. T47D and ZR-75-1 cells are human ER-positive breast carcinoma cell lines. T47D cells were used in our initial characterization of the IGF1R IRES (4) and in the highthroughput screen for small molecule IRES inhibitors (11). We subsequently found that MYC IRES was particularly sensitive to chemical inhibition in ZR-75-1 cells (11).

Population (standard) density assays were performed by seeding cells in full serum-containing medium, allowing $48 \mathrm{~h}$ for recovery, before initiating treatment with cells at $\sim 75 \%$ confluency ( 200,000 cells $\left./ \mathrm{cm}^{2}\right)$. Low (clonogenic)-density assays were performed by seeding cells in full serum medium at $\sim 700$ cells $/ \mathrm{cm}^{2}$ and allowing $48 \mathrm{~h}$ for recovery before initiating treatment. cpd_W was solubilized in $100 \%$ dimethyl sulphoxide (DMSO) to a concentration of $10 \mathrm{mg} / \mathrm{ml}$ and used immediately or stored at $-20^{\circ} \mathrm{C}$. Stock solutions were diluted a minimum of 1:1,000 in media until final DMSO concentration did not exceed $0.1 \%$, which was matched in vehicle-only control samples. The compound was thoroughly dispersed in media before being added to the cells. Low serum conditions (0.5\% FCS, no supplemental insulin) were used during treatment to simulate suboptimal microenvironmental conditions to which tumor cells are exposed in vivo and to increase dependence on IRES-mediated translation. For the washout and recovery assays, treatment was terminated by replacing with fresh media (without compound, but maintaining low serum conditions).

Indirect immunofluorescence staining and confocal imaging. The cells were seeded in 8-well chamber slides (Nunc; Nalge Nunc International, Penfield, NY, USA) and allowed $48 \mathrm{~h}$ to recover and resume proliferation prior to treatment with cpd_W or vehicle control $(0.1 \%$ DMSO) as indicated in the corresponding Figure legends. The cells were fixed with freshly prepared $2 \%$ paraformaldehyde for $15 \mathrm{~min}$ at room temperature, followed by permeabilization with $0.2 \%$ Triton X-100 for $10 \mathrm{~min}$. After washing in PBS with $75 \mathrm{mM}$ glycine and blocking in $5 \%$ normal goat serum, the primary antibody was added and incubated for $35 \mathrm{~min}$ to $1 \mathrm{~h}$ at room temperature. Following two washes in PBS and a 10 min reblocking step, secondary antibodies (1:200) were added and incubated for 25-45 min. Following two additional PBS washes, nuclei were stained with DAPI $(0.2 \mu \mathrm{g} / \mathrm{ml})$ and mounted using ProLong Gold (Life Technologies; Thermo Fischer Scientific).

Images were captured using a Nikon A1 confocal instrument with 40X 1.3 NA objective (Nikon Corporation, Tokyo, Japan). Fields were randomly selected for imaging on the basis of the DAPI staining pattern alone. Paired images of control and experimental wells were acquired sequentially and all settings including laser power, PMT voltage and pinhole were held constant between samples.

Cell viability assays. Viability was assessed based on ATP content (CellTiter-Glo; Promega Corporation, Madison, WI, USA). Appropriate negative controls (lysis buffer alone) were subtracted from the readings.

Western blot analysis. Whole cell lysates were prepared from treated cells as previously described (11) using lysis buffer containing 4\% SDS and $720 \mathrm{mM}$ 2-mercaptoethanol, pre-heated to $100^{\circ} \mathrm{C}$. For cells at population density, equivalent aliquots of protein from each sample were separated by SDS-PAGE, transferred to $0.2 \mu \mathrm{m}$ nitrocellulose membranes 
Table I. Primary antibodies used in the present study.

\begin{tabular}{|c|c|c|c|c|}
\hline Protein & Clone & Host & Source & Application (dilution) \\
\hline IGF1R & N20 & Rabbit & $\begin{array}{l}\text { Santa Cruz Biotechnology Inc. } \\
\text { (Dallas, TX, USA) }\end{array}$ & IF (1:67) \\
\hline IGF1R & $\mathrm{C} 20$ & Rabbit & Santa Cruz Biotechnology Inc. & WB $(1: 400)$ \\
\hline Myc & $\mathrm{N} 262$ & Rabbit & Santa Cruz Biotechnology Inc. & WB $(1: 400)$ \\
\hline RACK1 & 20 & Mouse IgM & BD Biosciences (Jan Jose, CA, USA) & WB $(1: 2,500)$, IF $(1: 100)$ \\
\hline $\mathrm{ER} \alpha$ & $\mathrm{D} 8 \mathrm{H} 8$ & Rabbit mono & $\begin{array}{l}\text { Cell Signaling Technology, Inc. } \\
\text { (Danvers, MA, USA) }\end{array}$ & WB $(1: 1,000)$, IF $(1: 500)$ \\
\hline GRP78 & 40 & Mouse & BD Biosciences & WB (1:500), IF (1:100) \\
\hline $\mathrm{ZO}-1$ & Mid & Rabbit & $\begin{array}{l}\text { Life Technologies; Thermo Fisher } \\
\text { Scientific (Waltham, MA, USA) }\end{array}$ & IF $(1: 100)$ \\
\hline E-cadherin & HECD1 & Mouse & $\begin{array}{l}\text { Invitrogen; Thermo Fisher Scientific } \\
\text { (Waltham, MA, USA) }\end{array}$ & WB $(1: 1,000)$, IF $(1: 200)$ \\
\hline CLIMP-63 & ab152154 & Rabbit & Abcam (Cambridge, MA, USA) & IF $(1: 160)$ \\
\hline $\mathrm{CHOP}$ & L63F7 & Mouse & Cell Signaling Technology, Inc. & WB $(1: 1,000)$ \\
\hline CDK1 & 17 & Mouse & Santa Cruz Biotechnology Inc. & WB $(1: 400)$ \\
\hline $\begin{array}{l}\text { Connexin } 43 \\
(\mathrm{Cx} 43)\end{array}$ & 3512 & Rabbit & Cell Signaling Technology, Inc. & WB $(1: 1,000)$ \\
\hline$\alpha$-tubulin & B-5-1-2 & Mouse & Sigma-Aldrich (St. Louis, MO, USA) & WB $(1: 4,000), \operatorname{IF}(1: 500)$ \\
\hline
\end{tabular}

mono, monoclonal; WB, western blotting; IF, immunofluorescence.

and probed with antibodies using standard immunoblotting procedures.

For western blot analysis of protein recovered from cells at clonogenic density, maximally-concentrated whole cell lysates were prepared by rapid serial transfer of a minimum volume $(100 \mu \mathrm{l})$ of lysis buffer to three adjacent replicate wells (each $10 \mathrm{~cm}^{2}$, standard 6-well plates). Western blot analysis of these samples was performed by loading equivalent aliquots (by volume) into each lane, without normalizing for total protein content.

In vitro translation. Standard in vitro translation reactions were set up using micrococcal nuclease treated rabbit reticulocyte lysate (Promega) at a final concentration of $50 \%$ (vol/vol). An intermediate aqueous dilution (1:50) of IRES inhibitor stock solution was prepared to allow for small volume addition to the translation reaction without excessive $(<0.2 \%$ final concentration) DMSO. Reactions were initiated by adding in vitro transcribed RNA ( $7 \mathrm{nM}$ final concentration) prepared from the bicistronic reporter construct pDualIGF1R(951-1040) containing the core functional IGF1R IRES. Reactions were incubated at $30^{\circ} \mathrm{C}$ for $100 \mathrm{~min}$ and firefly and Renilla luciferase activities were assessed using the standard dual luciferase assay protocol.

\section{Results}

Inhibition of IRES-mediated translation in vitro and in cells. IRES inhibitor lead compound W (cpd_W, Fig. 1B) was initially identified on the basis of its ability to selectively interfere with the expression of firefly luciferase from a bicistronic (IRES reporter) vector in breast tumor cells genetically engineered with this construct (11). As an even more specific test of mechanism of action, we titrated cpd_W in a cell-free in vitro translation assay, using rabbit reticulocyte lysate as a source of ribosomes, supplemented with amino acids and provided the bicistronic RNA which had been synthesized and purified in vitro. The results (Fig. 1C) indicated that cpd_W selectively inhibited the synthesis of firefly luciferase (product of the second cistron, mediated by the IRES) in a concentration-dependent manner, with no significant impact on the synthesis of Renilla luciferase (product of the first cistron, translated by conventional ribosome scanning). These results confirmed the mechanism of action in a simple reconstituted system in which potentially confounding factors such as transcription or signal transduction were not in play. The results also distinguished cpd_W from the universal inhibitors of protein synthesis cycloheximide or puromycin, which completely block translation of both cistrons.

In addition to its activity against the IRES reporter, it was critical that we determined whether cpd_W would also inhibit the function of the endogenous IGF1R IRES in untransfected cells. T47D (human ER-positive breast tumor) cells express IGF1R at a high level. To assess the impact of IRES inhibition on IGF1R protein level, the cells were incubated with cpd_W and simultaneously subjected to acute serum deprivation ( $0.5 \%$ FCS). Limiting access to soluble growth factors in this manner simulated the adverse microenvironmental conditions tumor cells are typically exposed to in vivo and caused cells to become more dependent on IRES-mediated translation. Following $24 \mathrm{~h}$ exposure to cpd_W, $50 \%$ decrease in IGF1R protein is observed (Fig. 1D, lanes 4 and 6). This relatively slow decline was consistent with the long half-life $(>24 \mathrm{~h})$ of pre-existing IGF1R protein molecules. 
A

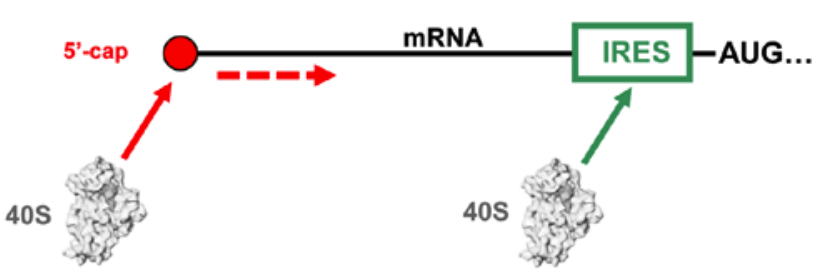

general protein synthesis

T mTOR inhibitors

IRES-mediated translation
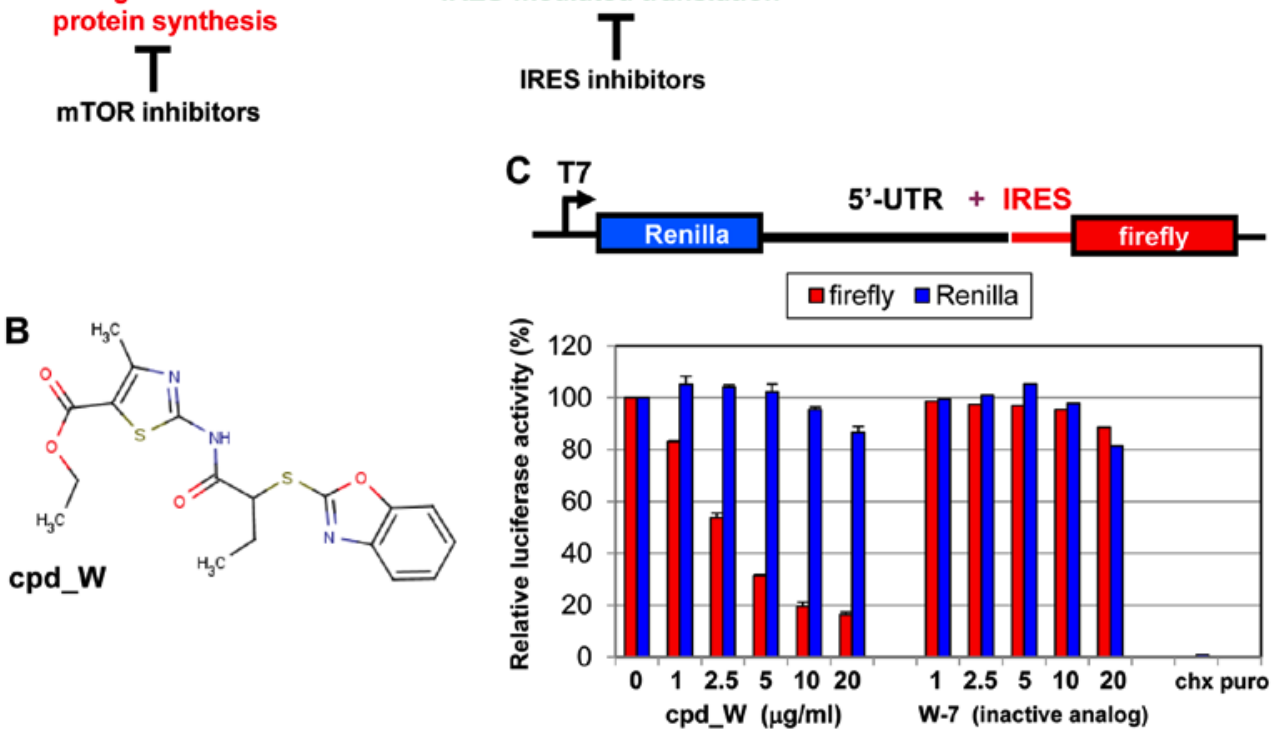

D

a, acute serum deprivation b, trypsin catabolism c, trypsin / suspension

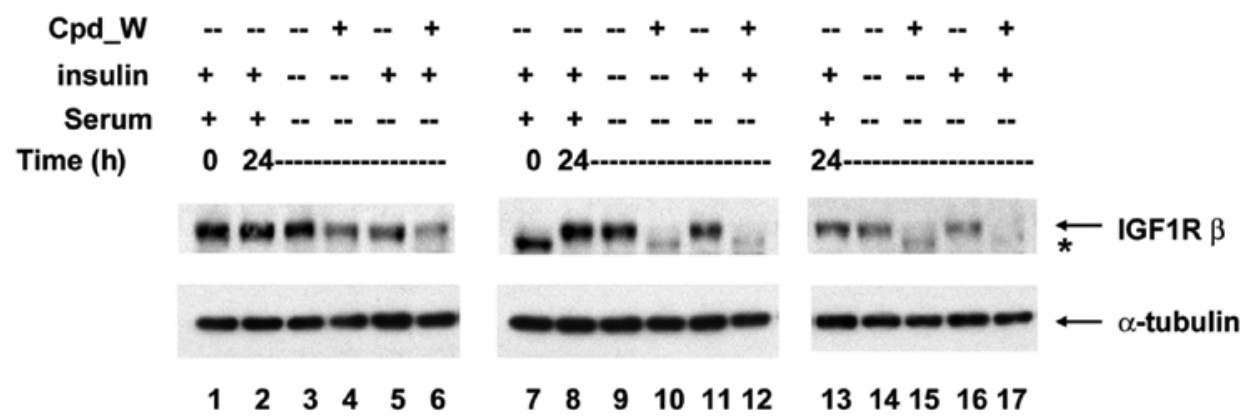

Figure 1. IRES-mediated translation and IRES inhibition in a cell-free system and in cells. (A) Diagrammatic comparison of general protein synthesis to IRES-mediated translation. General protein synthesis is mediated by cap-dependent ribosomal scanning from the 5'-end of the mRNA and may be modulated by mTOR inhibitors. Internal ribosome entry sites (IRESs) allow the 40S ribosome to engage the mRNA at a position much closer (in many cases immediately adjacent to) the AUG initiation codon. IRES-mediated translation is independently regulated and serves as a fail-safe mechanism ensuring the synthesis of proteins most critical for cell survival. (B) Structure of IRES inhibitor lead compound W (cpd_W): Ethyl 2-\{[2-(1,3-benzoxazol-2-ylthio)butanoyl]amino\}4-methyl-1,3-thiazole-5-carboxylate, MW 405. (C) In vitro translation assays: Rabbit reticulocyte lysate was programmed with a bicistronic reporter RNA in which translation of the second cistron (firefly luciferase coding sequence) is mediated by the IGF1R IRES, while translation of the first cistron (Renilla luciferase coding sequence) is mediated by ribosomal scanning. IRES inhibitor cpd_W (or vehicle control) was included in the reaction in increasing concentrations as indicated. The result is indicative of selective inhibition of IRES-mediated translation. A structural analog of cpd_W (W-7) in which a single atom has been modified (converting the benzoxazole to a benzimidazole) was completely inactive in this assay, indicative of the chemical specificity of IRES inhibition. Cycloheximide $(5 \mu \mathrm{g} / \mathrm{ml}$, chx) and puromycin $(250 \mu \mathrm{g} / \mathrm{ml}$, puro) were included as reference standards for non-specific translational inhibition (far right). (D) IRES inhibitor cpd_W completely blocked de novo synthesis of IGF1R in breast tumor cells under adverse conditions (serum-deprivation, loss of adhesion) relevant to the microenvironment of the tumor. T47D breast tumor cells were seeded in 6-well plates and allowed $48 \mathrm{~h}$ to recover and resume proliferation, then incubated in the presence of IRES inhibitor cpd_W $(10 \mu \mathrm{g} / \mathrm{ml})$ or vehicle control $(0.1 \%$ DMSO) as indicated. The cells were simultaneously subjected to acute serum deprivation ( $0.5 \%$ fetal calf serum, no added insulin) to increase dependence on IRES-mediated translation. After $24 \mathrm{~h}$, the cells were harvested and whole cell lysates prepared, equivalent aliquots separated by SDS-PAGE and immunoblotted for IGF1R- $\beta$ and $\alpha$-tubulin. In lanes 7-12, the cells were trypsinized and seeded into 6-well plates and immediately incubated in the presence of IRES-inhibitor cpd_W or vehicle control as indicated. Robust regeneration of trypsin-catabolized IGF1R was observed within $24 \mathrm{~h}$ in vehicle-treated cells, however, this was completely blocked in the presence of cpd_W (10 $\mu \mathrm{g} / \mathrm{ml}$ as shown; $\left.\mathrm{IC}_{50}, 2 \mu \mathrm{g} / \mathrm{ml}\right)$. The asterisk (") marks the position of trypsin-catabolized IGF1R. In lanes 13-17, the cells were treated as described for lanes 7-12, except that following trypsinization, cells were transferred to low-adherence plates, forcing cells to adapt to a state of anchorage-independence. The results confirmed the activity of cpd_W against the endogenous IRES in genetically-unmodified tumor cells. Similar results were obtained with IRES inhibitor lead cpd_P (11).

We found that the ability of the IRES inhibitor to block IGF1R synthesis could be more readily ascertained if the equilibrium was perturbed. When the cells were trypsinized (using the standard procedure for subculturing cells), all 
IGF1R molecules on the surface of the cell were digested, resulting in a complete shift (downward) on western blot analysis (Fig. 1D, lane 7). This forced the cells to regenerate the entire population of IGF1R molecules and provided an opportunity to clearly discriminate whether IGF1R synthesis would be inhibited by cpd_W. Robust regeneration of full-length IGF1R to baseline (pre-trypsin) level was observed within $24 \mathrm{~h}$ in vehicle-treated cells (lanes 8,9,11), however, this was completely blocked in the presence of cpd_W (lanes 10 and 12). Identical results were obtained when cells were simultaneously deprived of adhesion (forced anchorage-independence, lanes 13-17). These results confirmed the activity of cpd_W against the endogenous IGF1R IRES and matched the findings obtained with our first lead compound cpd_P (11).

ER-positive breast tumor cells exist in two major interconvertible phenotypic states. Subsequently, we developed imaging strategies which may allow us to directly monitor IRES-mediated translation in native (genetically-unmodified) cells. We anticipated that these imaging analyses may allow us to distinguish in which cells IRES-mediated translation is taking place and to correlate changes in phenotype with changes in translational activity at the level of the individual cell. Furthermore, we anticipated that such staining patterns may help to assess the consequences of IRES inhibition.

We first selected an antibody to IGF1R (N20) which recognizes the $\mathrm{N}$-terminal region of the protein in denatured form (i.e. on western blotting) but fails to detect the mature IGF1R protein at the cell surface. We reasoned that this antibody may recognize nascently-translated IGF1R at the rough endoplasmic reticulum where it is synthesized, prior to adoption of its native three-dimensional conformation and in this manner, may serve as an indicator of active IRES-mediated translation. At the same time, we stained the cells with an antibody to RACK1, which is an integral component of the $40 \mathrm{~S}$ ribosomal subunit, to mark the sites where active translation could take place. RACK1 resides near where interactions with the 5'-untranslated regulatory region of the mRNA take place (13) and is suspected of being involved in regulating IRES-mediated translation (14).

Applying this immunocytochemical staining strategy to the T47D ER-positive breast tumor cell line, two phenotypically distinct populations of cells were observed (Fig. 2A). One population of cells displayed high intensity staining for nascently-translated IGF1R, but very low intensity of RACK1 immunofluorescence. These cells tend to be arranged in clusters located within the interior of a mass of contiguous cells i.e. without exposed lateral surfaces. The other population of cells exhibited precisely the inverse pattern: High RACK1 intensity and very low staining for nascently-translated IGF1R. These cells were consistently found bordering open spaces, with concave lateral surfaces (planar polarity). The IGF1R N20 and RACK1 staining patterns were almost perfectly mutually exclusive, i.e. the majority of cells were either red or green, with essentially no overlapping.

At baseline, both phenotypes were present. However, if the cells were subjected to acute serum deprivation, over time a progressively larger proportion of cells adopted the IGF1R N20-positive/RACK1-negative (green) phenotype (Fig. 2B). In contrast, cells subjected to serum deprivation but simultaneously treated with the IRES inhibitor cpd_W exhibited just the opposite effect, with essentially all cells shifting to the IGF1R N20-negative/RACK1-positive (red) phenotype.

These findings suggested that the IGF1R N20-positive cells represent a relatively undifferentiated population of cells in which IRES-mediated translation is active, proliferating without evidence of a higher-ordered structural organization. Transition to the undifferentiated IRES-active phenotype could be expected to facilitate cell survival under challenging microenvironmental conditions. By contrast, the RACK1positive cells appeared to represent a more differentiated population of tumor cells, with planar polarity and growth restraint and little or no IRES activity. The apparent inverse correlation between RACK1 immunoreactivity and IRESmediated translation was unexpected. Western blot analysis revealed no significant change in RACK1 abundance under these conditions (Fig. 5). It rather appeared that the RACK1 epitope was masked when the ribosomes were actively engaged in IRES-mediated translation. The RACK1 immunoreactivity returned when IRES-mediated translation was curtailed in association with the physiological transition to the more differentiated phenotype, or when the IRES mechanism was rendered non-functional by the IRES inhibitor.

Early during the incubation with the IRES inhibitor, numerous cells were observed (circled in Fig. 2C) which contained discrete bright RACK1-positive foci in place of the diffuse RACK1 cytoplasmic staining pattern observed at later time-points or in control fields. These foci were present only transiently and only in cpd_W-treated cells, indicating that they arose as a direct consequence of IRES inhibition and may represent sequestered dysfunctional IRES-ribosome complexes. In addition, it is noteworthy that, while colocalization of RACK1 and IGF1R N20 was almost never observed, there were cells in the control fields (circled in Fig. 2D) exhibiting a biphenotypic pattern, in which the portion of the cytoplasm bordering an acellular space was stained distinctly positive for RACK1 (IRES-off), while the remainder of the cytoplasm (facing the interior of a cell cluster) stained positive for IGF1R N20 (IRES-active).

Additional biomarkers of the undifferentiated/IRES-active and differentiated/IRES-off phenotypes. Subsequently, it was important to identify additional markers of these two distinct phenotypes, in order to confirm our findings and further investigate the relationship between IRES-mediated translation and differentiation status in the ER-positive breast tumor cells. We examined additional antibodies to proteins relevant to translation and/or differentiation and found several which allowed these two phenotypic states to be readily distinguished.

Foremost among these was estrogen receptor a $(E R \alpha$, ESR1; Fig. 3A). Confocal images revealed that cytoplasmic localization of ER $\alpha$ correlated with the clustered/undifferentiated IRES-active phenotype, precisely mirroring the IGF1R N20 staining pattern and reciprocal to RACK1. It appeared that ER $\alpha$ was completely excluded from the nuclei of these undifferentiated cells, where it was prevented from transcriptionally programming cells to differentiate. IRES inhibition was accompanied not only by an increase in RACK1 immunoreactivity, but also by a marked decrease in overall intensity of ER $\alpha$ staining. ER $\alpha$ itself is known to be translated via an 
A

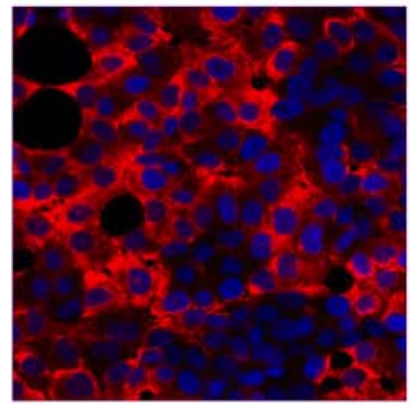

RACK1

B
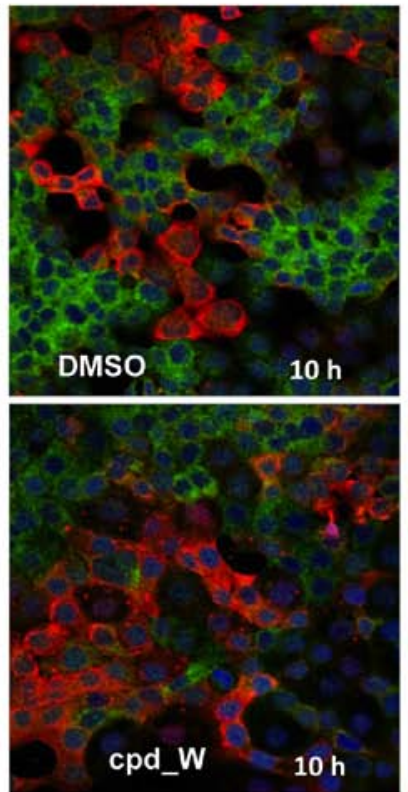

C

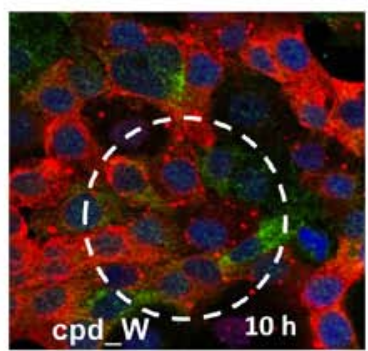

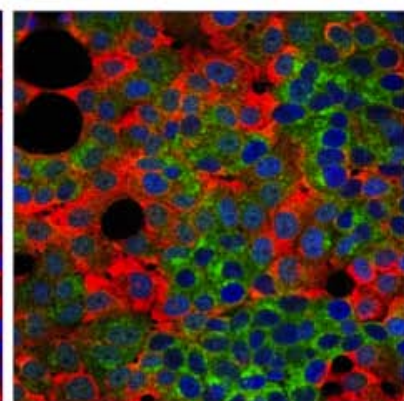

RACK1

IGF1R (N20)

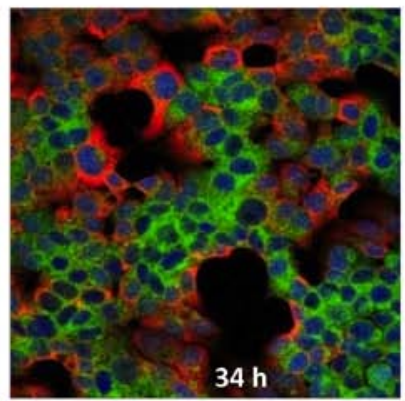

$34 \mathrm{~h}$
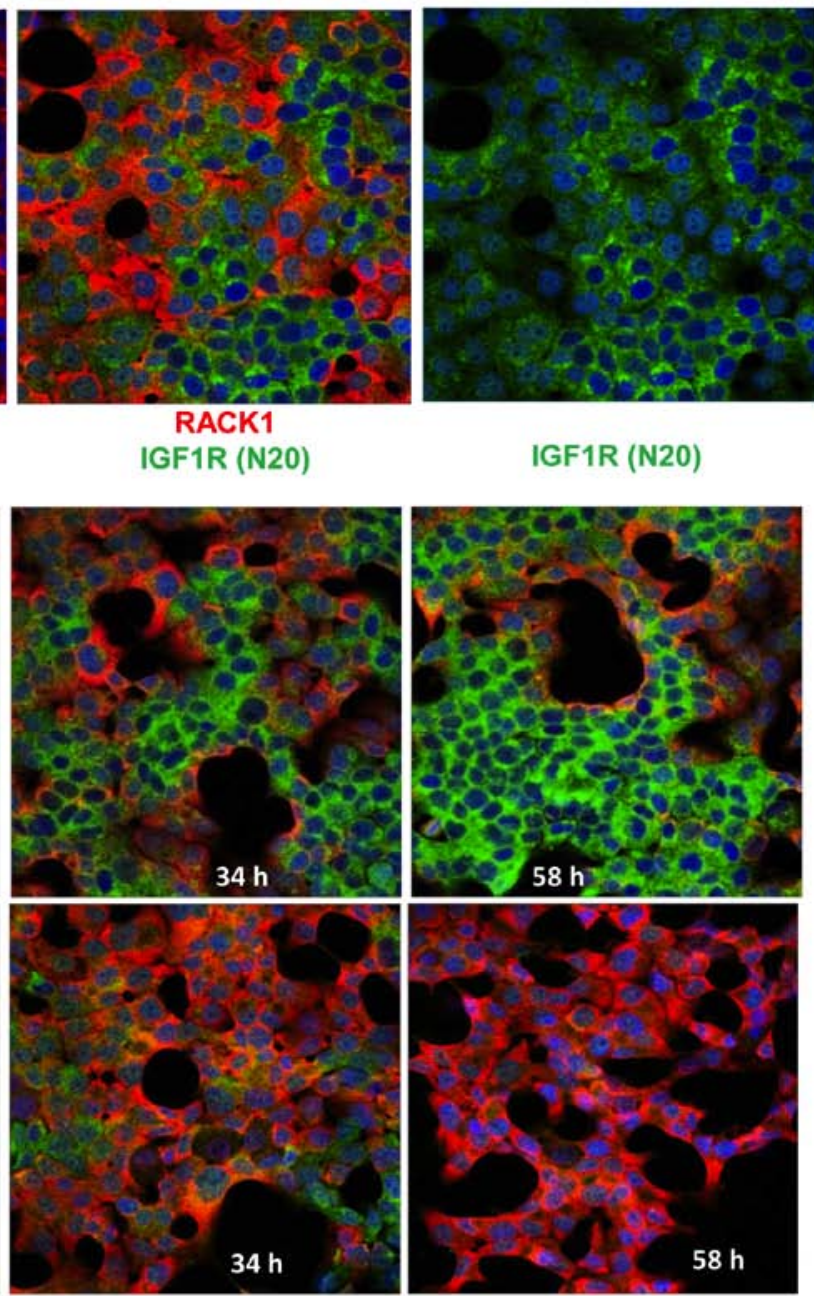

IGF1R (N20)
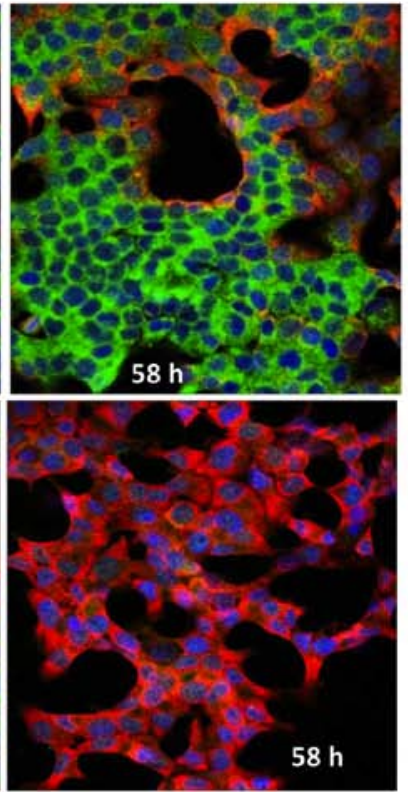

D

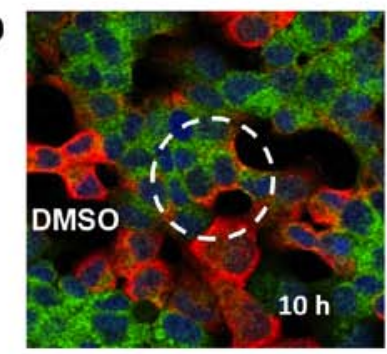

Figure 2. ER-positive breast tumor cell transition between two distinct phenotypic states distinguished by differentiation status and IRES-mediated translation. T47D breast tumor cells were seeded in 8-well chamber slides, allowed $48 \mathrm{~h}$ to recover and resume proliferation, then subjected to acute serum deprivation $(0.5 \%$ FCS $)$ in the presence of cpd_W at $10 \mu \mathrm{g} / \mathrm{ml}$ or vehicle control $(0.1 \%$ DMSO). At the indicated time-points, the cells were fixed, permeabilized and stained using N20 antibody to IGF1R and clone 20 antibody to RACK1, as described in the Materials and methods section. (A) Confocal images illustrating two distinct populations of tumor cells distinguished by mutually exclusive IGF1R N20 and RACK1 staining patterns. (B) RACK1/IGF1R N20 images of cells at 10,34 or $58 \mathrm{~h}$ time-points, demonstrated progressive phenotypic transitions. Cells subjected to serum deprivation (top row) became almost universally IGF1R N20-positive, while cells treated with IRES inhibitor cpd_W under the same conditions (bottom row) became entirely RACK1-positive. (C) Higher magnification image from cpd_W 10 h field in which cells bearing distinct cytoplasmic RACK1-positive foci (circled) were demonstrated. (D) Higher magnification image from DMSO $10 \mathrm{~h}$ field in which biphenotypic cells (circled) were demonstrated.

IRES (15), thus these results suggested that the ESR1 IRES may be sensitive to cpd_W.

The progressive transition of cells from the undifferentiated IRES-active (cytoplasmic ER $\alpha$-positive) phenotype to the differentiated IRES-off (RACK1-positive) state is displayed in Fig. 3B, where the parallel gain in structural organization, with the cells distinctively arranged to surround open spaces, is also evident. With-24 h exposure to the IRES inhibitor, the majority of cells became RACK1-positive and ER $\alpha$ had shifted almost entirely to the nucleus. Following $48 \mathrm{~h}$ continuous IRES inhibition, only a small quantity of ER $\alpha$ remained. A similar pattern, including shifting of ER $\alpha$ to the nucleus upon differentiation, was observed in another ER-positive breast tumor cell line, ZR-75-1 (Fig. 3C).

GRP78 (HSPA5, BiP) is a prosurvival molecule integrally involved in translation quality control. GRP78 nuclear immunoreactivity exhibited a strong correlation with the differentiated IRES-off state (Fig. 3D and E). While the vehicle-treated field 
A
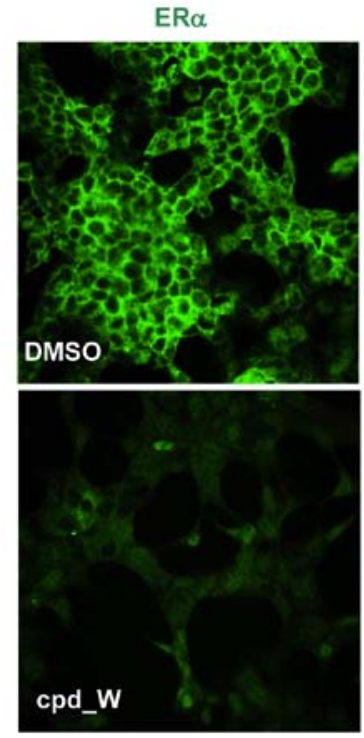

B

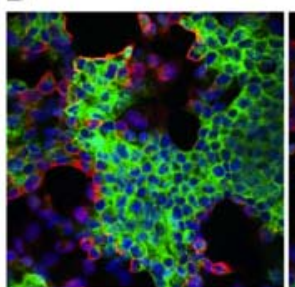

DMSO

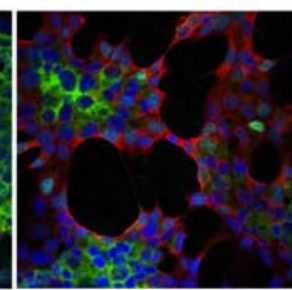

cpd_W X 15 h
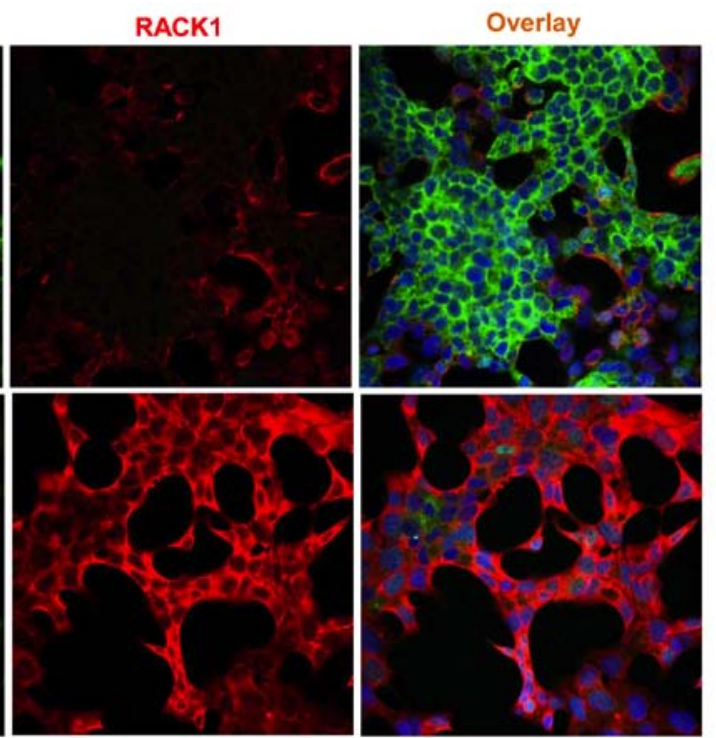

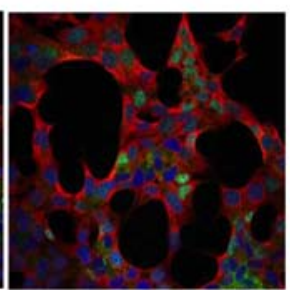

cpd_W X 24 h

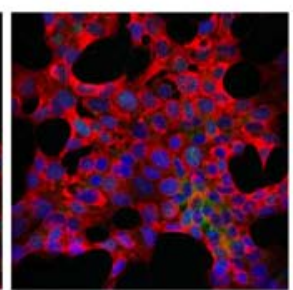

cpd_W X 48 h

Figure 3. Biomarkers of the undifferentiated/IRES-active and differentiated/IRES-off phenotypes. (A) T47D cells were treated with IRES inhibitor cpd_W as described in Fig. 2. At the $48 \mathrm{~h}$ time-point, the cells were fixed and stained for ER $\alpha$ (D8H8) and RACK1. (B) T47D cells were treated with cpd_W for 15, 24 or 48 h. Exposure to cpd_W was terminated by replacing with fresh media and cells were fixed and stained for ER $\alpha$ and RACK1 at the 48 h time-point.

included undifferentiated IGF1R N20-positive cells in which nuclear GRP78 was not observed, following treatment with cpd_W, the cells became uniformly negative for IGF1R N20 and positive for nuclear GRP78. De novo appearance of the tight junction protein ZO-1 within the nuclei of the cpd_W-treated cells was also observed (Fig. 3F and G). The translocation of all three of these molecules: ER $\alpha$, GRP78 and ZO-1 to the nucleus is consistent with the nuclear (transcriptional) dominance of the differentiated state.

The images displayed in Fig. 3H confirmed that RACK1 colocalized extensively with CLIMP-63 (CKAP4), an established marker of the rough endoplasmic reticulum $(16,17)$, consistent with its tight association with ribosomes and confirming that RACK1 staining (when its epitope is not masked) marked the sites where translation could take place. Notably this is the only pair of antibodies used in the present studies for which any significant degree of colocalization was observed, further highlighting the mutual exclusivity of the two distinct phenotypes displayed by the ER-positive breast tumor cells.

Marked changes in the distribution of E-cadherin and reorganization of the $\alpha$-tubulin cytoskeleton were observed accompanying the differentiation induced by the inhibition of IRES-mediated translation (Fig. 3I and J). Notably, these phenotypic changes persisted even after the compound was removed from the media, indicating that a finite period during which synthesis of critical IRES-driven proteins was blocked may have a long-lasting impact on the phenotype of those cells.
ER-positive breast tumor cells tolerate forced differentiation however reproductive capacity is severely compromised by IRES inhibition. Although marked phenotypic changes were observed in the ER-positive breast tumor cells subjected to IRES inhibition, it did not appear that cell viability was markedly affected. To document this, cell viability assays were performed on cells following $96 \mathrm{~h}$ exposure to cpd_W at varying concentrations ranging from $<1$ to $10 \mu \mathrm{g} / \mathrm{ml}$. The results (black lines) obtained for both T47D (Fig. 4A) and ZR-75-1 cell lines (Fig. 4B) indicated that these cells tolerated prolonged IRES inhibition with relatively modest decreases in the number of viable cells.

Subsequently we examined whether the forced transition to the differentiated phenotype brought about by the inhibition of IRES-mediated translation may be irreversible, i.e. whether the tumor cells may be hindered in their ability to transition back to the undifferentiated state and resume proliferation. To examine whether the reproductive capacity of the breast tumor cells subjected to IRES inhibition may have been altered, secondary clonogenic survival assays were performed. Following 96-h treatment and 24-h recovery period in the absence of compound, the cells were trypsinized and reseeded at low density. The cells were provided a 6-day period to proliferate (in the absence of compound) and then cell viability was assessed (Fig. 4A and B; purple lines). The cells which had been treated with cpd_W at $<2.5 \mu \mathrm{g} / \mathrm{ml}$ demonstrated a repopulating ability that very closely matched the viability readout assessed immediately after treatment (i.e. 
C
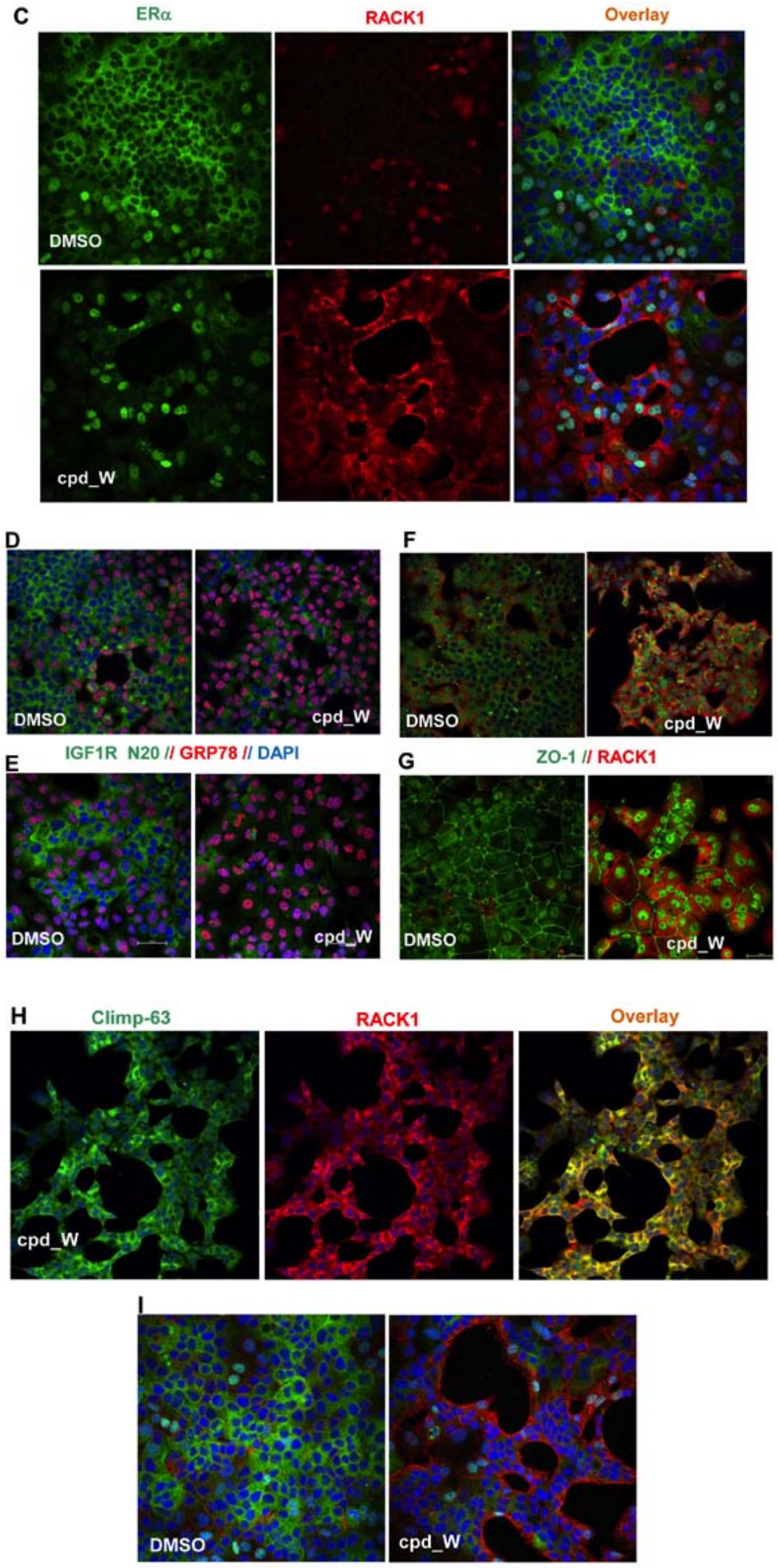

ER $\alpha$ // E-cadherin // DAPI

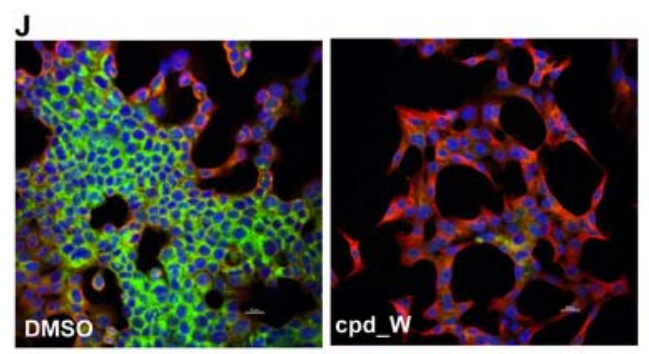

IGF1R N20 // $\alpha$-tubulin // DAPI

Figure 3. Continued. (C) ZR-75-1 cells were treated and stained for ER $\alpha$ and RACK1 as abovedescribed in (A). (D-J) T47D (D, F, H and J) or ZR-75-1 (E, G and I) cells were treated with cpd_W or vehicle control for $48 \mathrm{~h}$, then stained for IGF1R N20 and GRP78 (D and E), RACK1 and ZO-1 (F and G), RACK1 and CLIMP-63 (H), ER $\alpha$ and E-cadherin (I), or IGF1R N20 and $\alpha$-tubulin (J). 

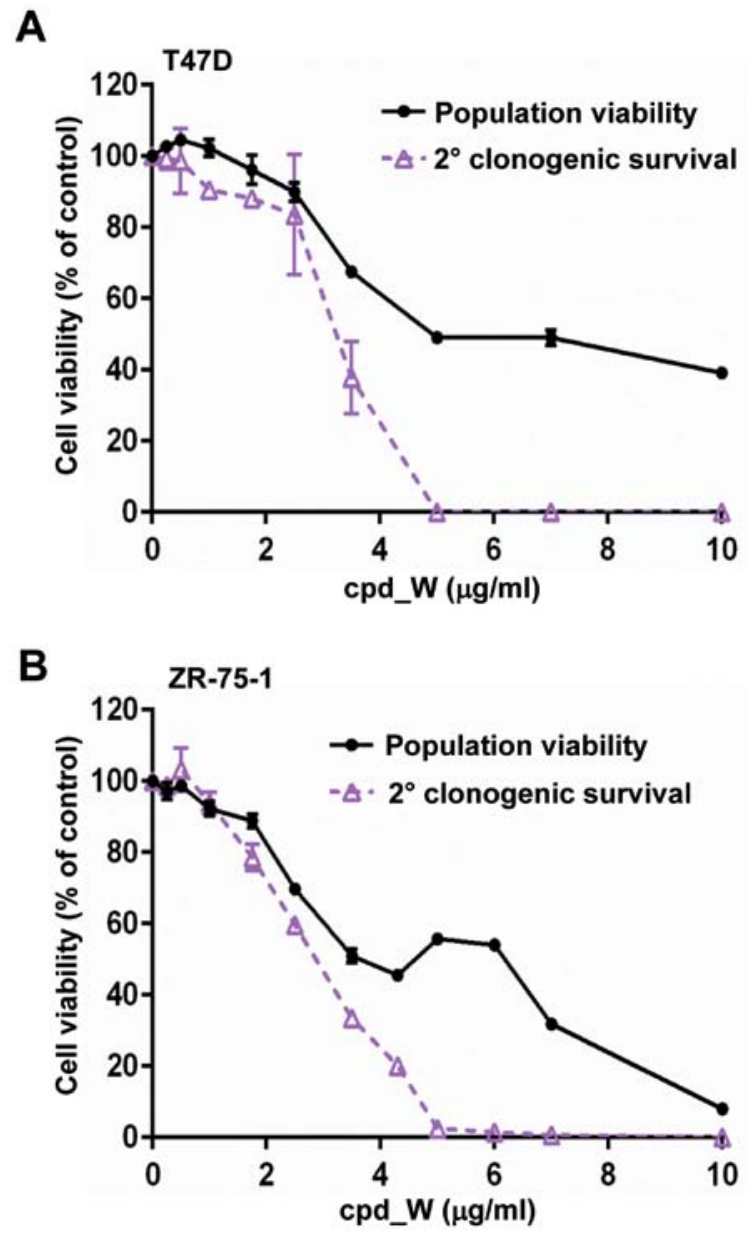

Figure 4. Loss of reproductive capacity in ER-positive breast tumor cells as a consequence of inhibition of IRES-mediated translation. T47D (A) or ZR-75-1 (B) cells $~ 75 \%$ confluent were treated with IRES inhibitor cpd_W at concentrations ranging from $0.25-10 \mu \mathrm{g} / \mathrm{ml}$ for $96 \mathrm{~h}$, after which the viability was assessed based on ATP content as described in Materials and methods section. The results (population viability, black lines) are plotted relative to vehicle control. In parallel, a set of identically prepared samples, following $96 \mathrm{~h}$ treatment, was provided fresh media (containing full serum) and allowed to recover for $24 \mathrm{~h}$, then trypsinized and reseeded at low density, allowed 6 days to proliferate, then viability assessed as a measure of reproductive capacity (secondary clonogenic survival, purple lines).

minimal separation of the black and purple curves). However, a sharp decline in the reproductive capacity was observed at $3.5 \mu \mathrm{g} / \mathrm{ml}$ cpd_W and for the cells which had been treated with $>5 \mu \mathrm{g} / \mathrm{ml}$ cpd_W, the ability to repopulate the well was almost completely lost $(<2.5 \%$ of control). The same pattern was observed in both T47D and ZR-75-1 cell lines. Thus, the secondary clonogenic survival assay revealed the severe detrimental impact of IRES inhibition on the ER-positive breast tumor cell population, which was not readily apparent from the initial viability readout. In essence, these cells were metabolically alive, yet reproductively dead at the end of the treatment period, consistent with terminal differentiation.

Alterations to critical phenotype-determining proteins in ER-positive breast tumor cells subjected to IRES inhibition. A series of western blot analyses were performed to examine changes taking place in abundance of individual proteins as a consequence of IRES inhibition (Fig. 5A and B). Although the long half-life of pre-existing IGF1R molecules precluded a more rapid or more substantial impact, a modest decrease in IGF1R is observed in cells treated with $>5 \mu \mathrm{g} / \mathrm{ml} \mathrm{cpd} \_W$ (consistent with the results of Fig. 1). A marked decrease in Myc protein brought about by IRES inhibition was observed in the ZR-75-1 cells, which expressed Myc at a high level. In addition, ER $\alpha$, CDK1 and connexin 43, each of which is known to be translated via an IRES $(15,18,19)$, were decreased substantially in a concentration-dependent manner upon exposure to cpd_W.

The loss of ER $\alpha$ protein from the treated cells was consistent with the imaging results. Notably, there are two dynamics in play for ER $\alpha$ : i) a shift from the cytoplasm to the nucleus in association with phenotypic transition to the differentiated phenotype; and ii) a decrease in the synthesis of ER $\alpha$ protein as a result of the inhibition of IRES-mediated translation. Likewise, CDK1 levels declined rapidly, especially in T47D cells and connexin 43 essentially disappeared from the treated cells within $24 \mathrm{~h}$. In contrast, a marked induction of both GRP78 and CHOP was observed in response to IRES inhibition, consistent with the involvement of these molecules in the translation quality control.

$\mathrm{ER} \alpha, \mathrm{CDK} 1$ and connexin 43 were actively being translated at the time the IRES inhibitor was added and the synthesis of these proteins did not rapidly rebound when the IRES inhibitor was removed from the media (though there was evidence of recovery of ER $\alpha$ during the washout period in ZR-75-1 cells following treatment with $5 \mu \mathrm{g} / \mathrm{ml} \mathrm{cpd \_ W).}$ This suggests that either these mRNAs remained sequestered in dysfunctional IRES-ribosome complexes, or that molecular changes induced during the period in which IRES-mediated translation was inhibited altered the phenotype so significantly that IRES-mediated translation of these proteins was no longer a priority for these cells. Notably, GRP78 remained elevated during washout, however CHOP dissipated quite rapidly following the removal of the IRES inhibitor. For RACK1 and E-cadherin, in spite of major changes in epitope accessibility and structural organization respectively, no significant alterations were observed in abundance of either of these proteins.

ER-positive breast tumor cells at low density (with limited paracrine support and no intercellular contact) exhibit enhanced susceptibility to IRES inhibition. Primary clonogenic survival assays revealed a significant decrease in colony formation at $0.1-0.25 \mu \mathrm{g} / \mathrm{ml} \mathrm{cpd} \_\mathrm{W}$ and almost complete loss of colony formation at $>0.5 \mu \mathrm{g} / \mathrm{ml}$ cpd_W (Fig. 6A). This was a much lower concentration of cpd_W than required to eliminate reproductive capacity of the high-density tumor cell population, indicating that sensitivity to IRES inhibition was enhanced for isolated or low-density ER-positive breast tumor cells. To follow up on this observation, a series of such experiments was performed using cell viability readouts as a quantitative surrogate for colony formation. The primary clonogenic survival dose-response curves for both T47D and ZR-75-1 (Fig. 6B and C; green lines) were superimposed on the population viability and secondary clonogenic survival curves from Fig. 4. The marked increase in susceptibility to IRES inhibition for the cells treated at low density was apparent from the wide separation between these curves. In fact, the viability readout tended to underestimate the negative impact of IRES inhibition on clonogenic survival, as many of the cells 
A

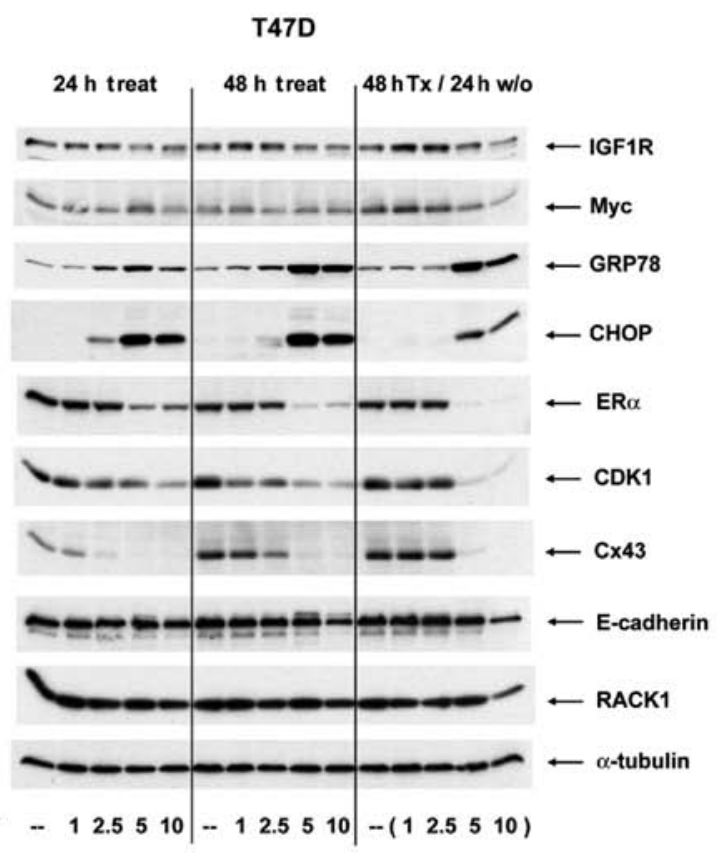

B

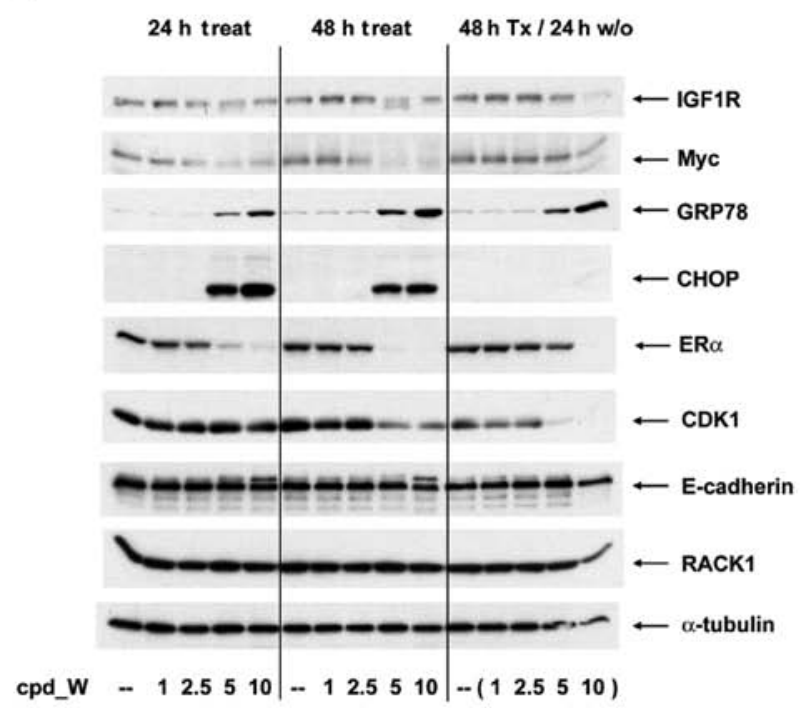

Figure 5. Impact of IRES inhibition on IGF1R, Myc, ER $\alpha, \mathrm{CDK} 1$ and connexin 43 (Cx43). T47D (A) or ZR-75-1 (B) cells $\sim 75 \%$ confluent were treated with varying concentrations of IRES inhibitor cpd_W or vehicle control as indicated for 24 or $48 \mathrm{~h}$, or $48 \mathrm{~h}$ followed by a $24 \mathrm{~h}$ washout period in media with no compound added. Whole cell lysates were prepared, equivalent aliquots separated by SDS-PAGE and used for western blot analyses of the indicated proteins.

remaining in the treated wells and scoring as viable based on ATP content were visualized microscopically as individual or pairs of cells, apparently unable to generate colonies even following prolonged incubation in absence of compound.

Thus, the ER-positive breast tumor cells responded to IRES inhibition very differently when isolated or present at low density compared to their response as part of a high-density population. Both the nature of the response and quantitative outcome were different. The cells at high density undergo terminal differentiation when IRES-mediated translation was inhibited, however at low density, where cells depend on
A

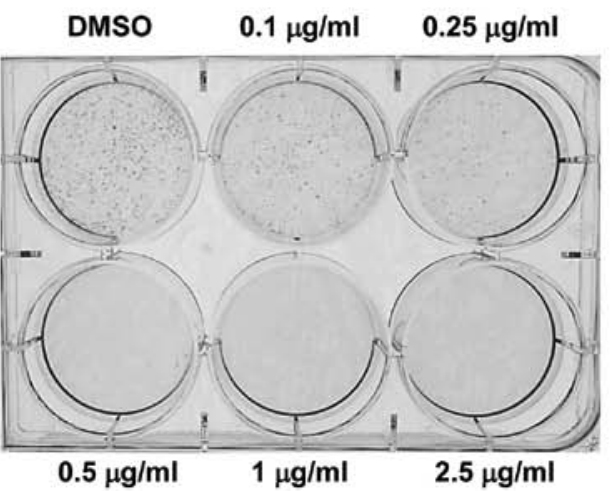

B

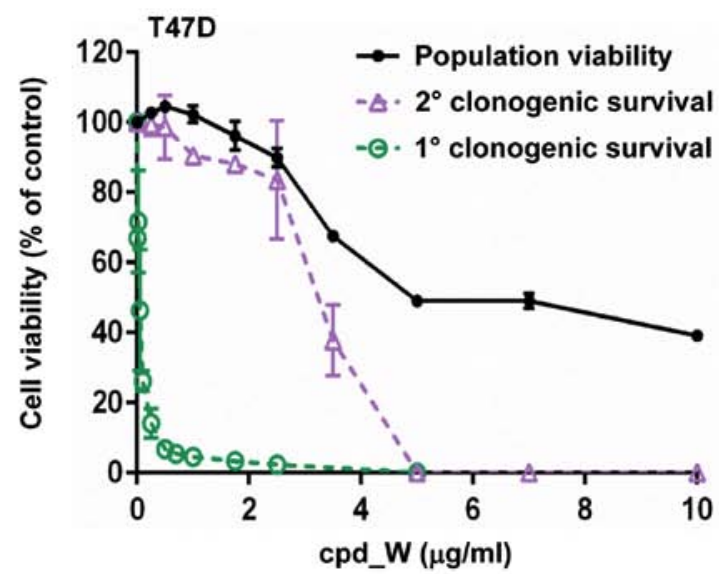

C

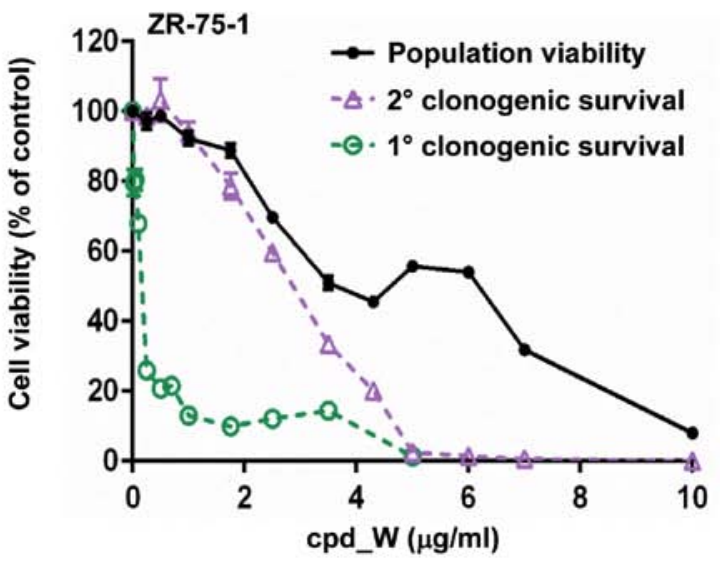

Figure 6. Loss of clonogenic survival in ER-positive breast tumor cells subjected to inhibition of IRES-mediated translation. (A) T47D cells were seeded at low (clonogenic) density $\left(\sim 700 \mathrm{cells} / \mathrm{cm}^{2}\right)$, allowed $48 \mathrm{~h}$ to recover and then treated with IRES inhibitor cpd_W at increasing concentrations as indicated for $96 \mathrm{~h}$ under low serum $(0.5 \% \mathrm{FCS})$ conditions, followed by 11 days of incubation in absence of compound. Colonies were stained with MTT to enhance visualization. T47D (B) or ZR-75-1 (C) cells were seeded at clonogenic density in 24 well plates, allowed $48 \mathrm{~h}$ to recover, then treated with cpd_W (or vehicle control, DMSO 0.1\%) under low serum conditions for $96 \mathrm{~h}$, after which fresh media was provided and the cells were allowed 9-10 days to proliferate. Cell viability was assessed and used as a quantitative surrogate for colony formation. The results (primary clonogenic survival, green lines) are superimposed on the graphs from Fig. 4, to facilitate direct comparisons. The use of ATP-based assay tended to underestimate the negative impact of IRES inhibition on clonogenic survival, since cells may register as viable yet not be capable of generating a colony.

IRES-mediated translation and the undifferentiated phenotype to survive, IRES inhibition led to acute cell death. 

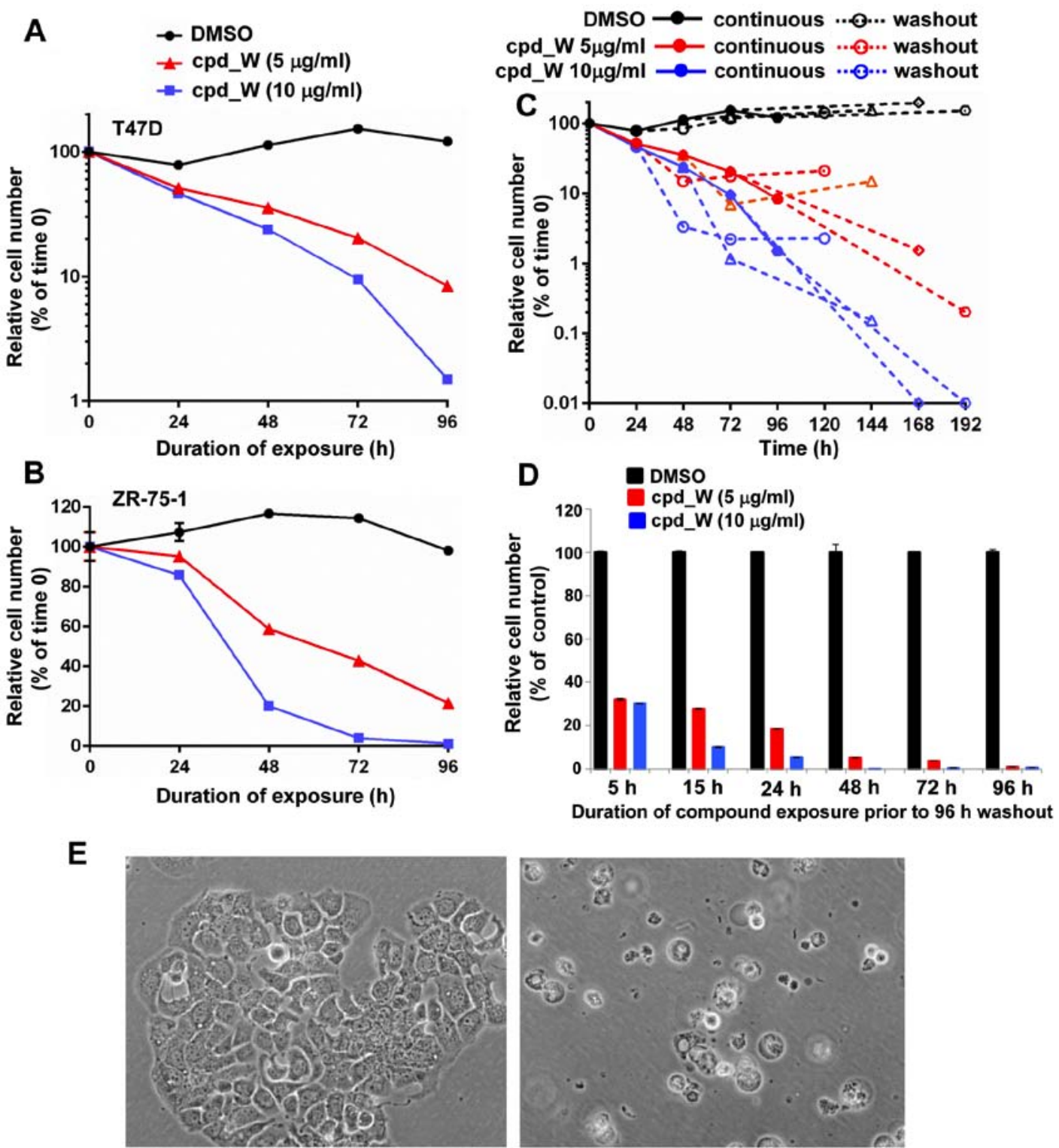

DMSO

cpd_W $5 \mu \mathrm{g} / \mathrm{ml}$

Figure 7. Massive cell death as a consequence of IRES inhibition in low-density ER-positive breast tumor cells. T47D (A) or ZR-75-1 (B) cells were plated at clonogenic density, allowed $48 \mathrm{~h}$ to recover, then treated with IRES inhibitor cpd_W at 5 or $10 \mu \mathrm{g} / \mathrm{ml}$ or vehicle control under low serum conditions for up to $96 \mathrm{~h}$ and then viability was assessed at $24 \mathrm{~h}$ intervals. (C) T47D cells were seeded at clonogenic density then treated with cpd_W at $5 \mu \mathrm{g} / \mathrm{ml}$ (red lines) or $10 \mu \mathrm{g} / \mathrm{ml}$ (blue lines) or vehicle (DMSO) control (black lines) for 24, 48,72 or $96 \mathrm{~h}$. The cells were then provided fresh media without compound and allowed $96 \mathrm{~h}$ to recover and proliferate before viability was again assessed. Solid symbols and lines are indicative of continuous treatment, while open symbols and dashed lines are indicative of washout period. The trajectories of the dashed lines allowed us to gauge the impact of IRES inhibition on cell survival following removal of the compound. (D) ZR-75-1 cells were seeded at clonogenic density then treated with cpd_W at 5 or $10 \mu \mathrm{g} / \mathrm{ml}$ for $5,15,24,48,72$ or $96 \mathrm{~h}$, after which all samples were provided fresh media and allowed $96 \mathrm{~h}$ to recover in the absence of compound. The graph plotted relative viability at the end of the $96 \mathrm{~h}$ washout period. (E) Phase contrast images of T47D cells treated at low density with cpd_W at $5 \mu \mathrm{g} / \mathrm{ml}$ for $96 \mathrm{~h}$.

The time course assays presented in Fig. 7A and B revealed the progressive loss of viability in these cells, reaching $80-90 \%$ cell loss by $96 \mathrm{~h}$ with $5 \mu \mathrm{g} / \mathrm{ml}$ cpd_W and $>98 \%$ cell loss at $10 \mu \mathrm{g} / \mathrm{ml}$ cpd_W. Fig. $7 \mathrm{C}$ is a plot of the trajectories of cell survival when the duration of exposure to cpd_W was titrated and cell viability was assayed $96 \mathrm{~h}$ following removal of the compound. Cells treated for $>72 \mathrm{~h}$ uniformly exhibited a continued downward trend in viability after the compound was removed from the media. In fact, cell numbers declined an additional 1-2 logs during the washout period, indicating that the detrimental impact of IRES inhibition persisted well beyond the treatment period. The results obtained for low-density ZR-75-1 cells (Fig. 7D) indicated that as little as 5-15 h interruption in IRES-mediated translation had a lasting impact on the proliferative capacity of the cells, but $72-96 \mathrm{~h}$ continuous exposure to cpd_W was required to ensure that the loss of cell viability was extensive and cell recovery was minimal. Phase contrast images illustrated the predominant morphology exhibited by low-density cells succumbing to IRES inhibition, characterized by gross osmotic swelling and widespread detachment (Fig. 7E), very different from the morphology observed with terminal differentiation at high cell density (Fig. 3) (12). 
A

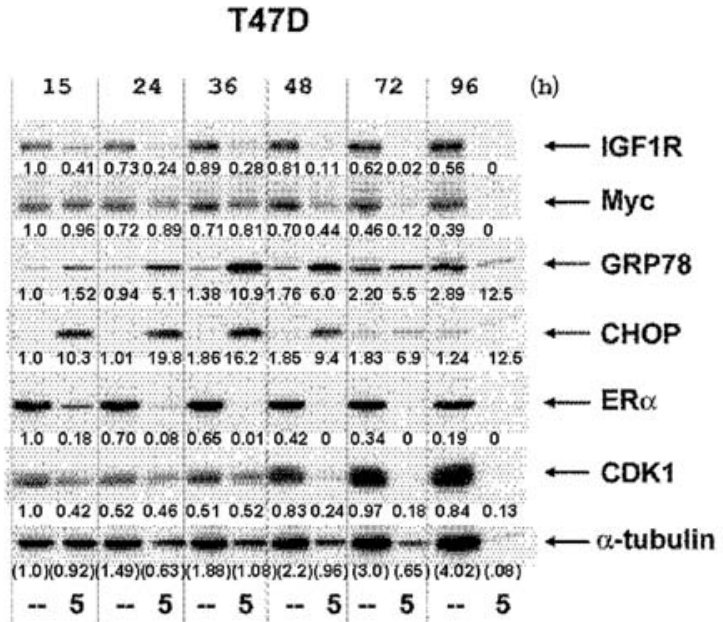

B

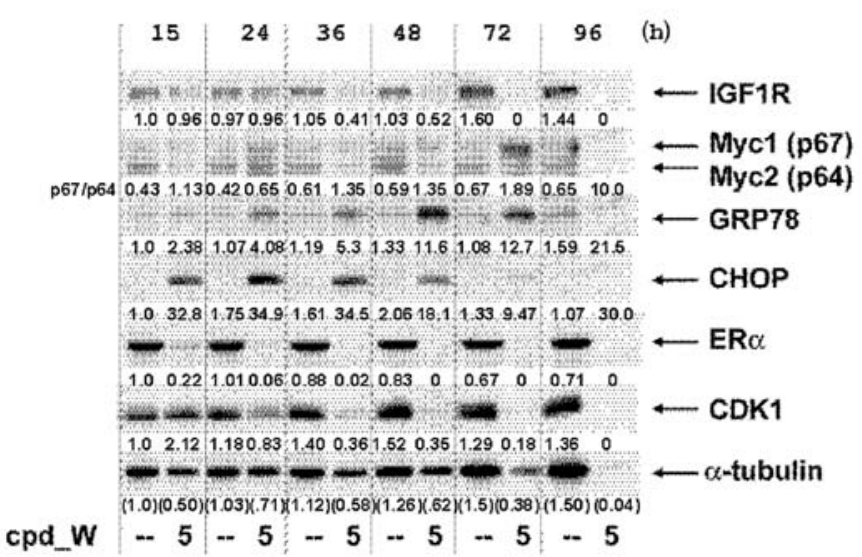

Figure 8. Enhanced sensitivity of IGF1R, Myc, ER $\alpha$ and CDK1 to IRES inhibition in low-density ER-positive breast tumor cells. T47D (A) or ZR-75-1 (B) cells were seeded at clonogenic density in groups of three replicate $10 \mathrm{~cm}^{2}$ wells and treated with cpd_W at $5 \mu \mathrm{g} / \mathrm{ml}$ or vehicle control under low serum conditions for up to $96 \mathrm{~h}$. Whole cell lysates were combined from three wells of each condition at each time-point. Equivalent aliquots (by volume, not protein content) were electrophoresed on 10 or $13 \%$ polyacrylamide gels and used for western blot analysis of the indicated proteins. Variations in intensity of $\alpha$-tubulin bands are indicative of changes in viable cell number during the course of the experiment. The light grey vertical lines placed over the ECL images were included only for orientation; the 12 lanes are contiguous. The numbers superimposed below each band are indicative of relative intensity as determined by quantitative densitometry. Values for $\alpha$-tubulin (in parentheses) were expressed relative to the vehicle control at the earliest time-point. Values for other proteins were normalized to the abundance of $\alpha$-tubulin in each sample. Values for Myc in ZR-75-1 cells represented the ratio of intensities of the p67 and p64 isoforms, which were reversed as a consequence of treatment with the IRES inhibitor.

Western blot analyses were performed on low (clonogenic) density cells by pooling lysates obtained from multiple wells, as described in the Materials and methods section. We deliberately elected not to normalize for total protein content in these very limited samples, but instead loaded a constant proportion (by volume) of the material recovered from the control and treated wells (Fig. 8A and B). The progressive decrease in the number of viable cells from cpd_W-treated wells (vs. the expansion of the vehicle-treated control cells) could be assessed by the relative intensities of the $\alpha$-tubulin bands and fitted well with the quantitative cell viability data presented in the abovementioned graphs. However, the intensities of bands representing IRES-driven proteins declined even more rapidly as a consequence of the inhibition of IRES-mediated translation and more rapidly than they did in the standard western blot analyses performed on high-density tumor cells. ER $\alpha$ declined to less than half the intensity of the control cells by $15 \mathrm{~h}$ and was barely detectable by $24 \mathrm{~h}$. Notable decreases in IGF1R and CDK1 were observed in both cell lines, with only low levels remaining at or beyond the 36 and $48 \mathrm{~h}$ time-points. Changes in Myc protein were accentuated in ZR-75-1 cells, with a pronounced induction of the alternative isoform of Myc (p67), particularly at the $72 \mathrm{~h}$ time-point, as cell death was accelerating. The dominant isoform of Myc (p64) is a major contributor to breast oncogenesis (20). However, the p67 isoform of Myc, which is known to be generated via use of an alternative upstream initiation codon, has been attributed potent growth inhibitory and pro-death properties $(21,22)$ and was also observed in the triple-negative breast tumor cells subjected to IRES inhibition, where it correlated with terminal differentiation and comprehensive death of those cells $(11,12)$. These decreases in IGF1R, CDK1 and ER $\alpha$ and the modulation of Myc translation (including shift to the p67 isoform) may be key molecular events that contributed to the stochastic death of low-density tumor cells in which IRES-mediated translation has been inhibited.

Incapacitation of low-density ER-positive breast tumor cells treated with very low concentrations of IRES inhibitor $c p d \_W$. Finally, we further characterized the impact of very low concentrations of cpd_W on the ER-positive breast tumor cells, in an attempt to understand the basis for clonogenic survival being so exquisitely sensitive to IRES inhibition. Time course assays indicated that exposure of low-density ER-positive breast tumor cells to a very low concentration $(1 \mu \mathrm{g} / \mathrm{ml})$ of cpd_W did not result in rapid or massive cell death (Fig. 9A). In fact the cell number was relatively stable throughout the $96 \mathrm{~h}$ treatment period, as cells surviving with limited paracrine support and low soluble growth factors attempted to proliferate and generate colonies. A gradual separation of the curves between vehicle control and cpd_W-treated samples was observed over the course of the experiment, with much of this separation occurring after the compound had been removed from the media. At the final endpoint, the vehicle controls have established viable colonies and were accelerating proliferation, while the cells which had been exposed to the IRES inhibitor at the beginning of the experiment continued to exhibit a slow progressive decline in cell number and few or no viable multi-cellular colonies.

Phase contrast images captured in the course of these experiments revealed that, although the cells exposed to $1 \mu \mathrm{g} / \mathrm{ml}$ cpd_W retain the ability to divide, the cells produced were dysmorphic and lacked the cohesiveness typical of ER-positive breast tumor cells (Fig. 9B). The cpd_W-treated cells exhibited very limited intercellular contact, in stark contrast to the extensively interconnected cobblestone morphology displayed by the control cells. Collectively, these findings indicated that the potent inhibition of clonogenic survival by very low concentrations of the IRES inhibitor resulted not from acute cell death, but rather from incapacitation, i.e. inability to 
A

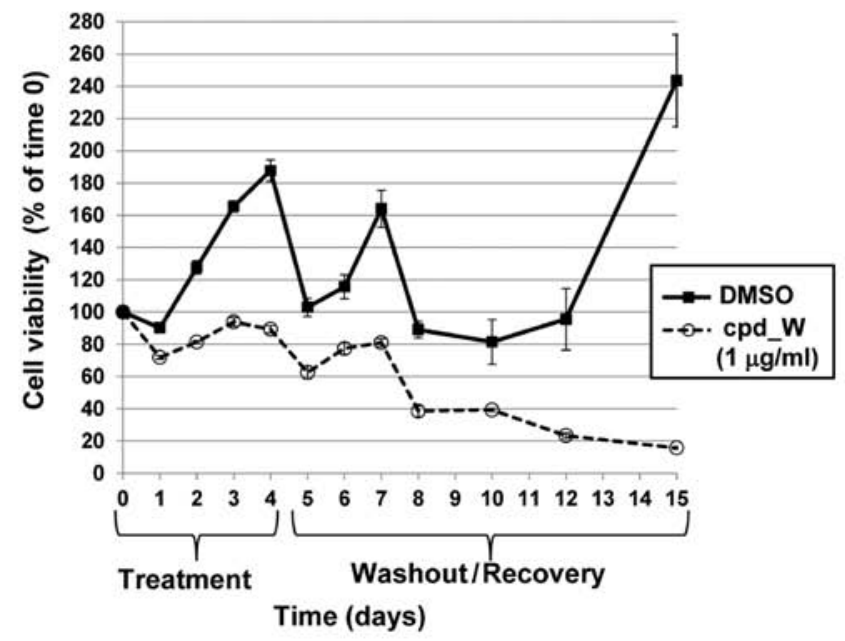

B

T47D $96 \mathrm{~h}$

DMSO
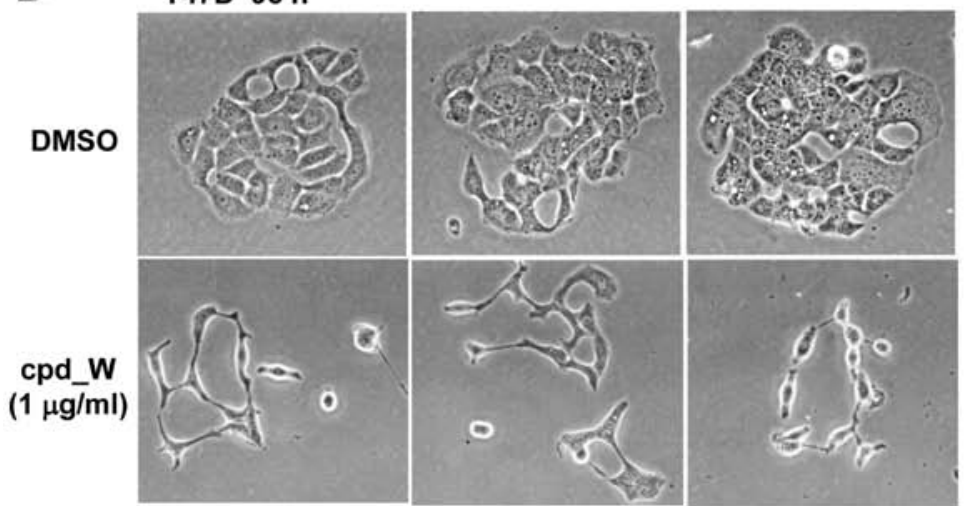

C

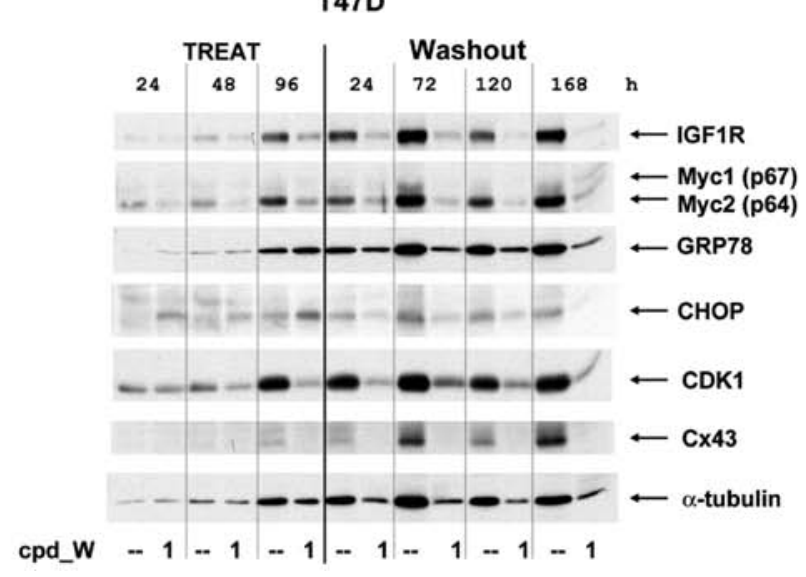

Figure 9. ER-positive breast tumor cells lose the ability to generate viable cohesive colonies when exposed to very low concentrations of IRES inhibitor cpd_W. (A) T47D cells were seeded at clonogenic density, treated for $96 \mathrm{~h}$ with cpd_W at $1 \mu \mathrm{g} / \mathrm{ml}$ or vehicle control $(0.01 \%$ DMSO), then provided fresh media and allowed 11 days for recovery. Low serum conditions were maintained throughout the experiment. The viability was assessed periodically on replicate wells throughout the course of the experiment. (B) Phase contrast images captured following $96 \mathrm{~h}$ treatment of low-density T47D cells with $1 \mu \mathrm{g} / \mathrm{ml} \mathrm{cpd} \_\mathrm{W}$ revealed the defect in colony formation induced by very low concentration of the IRES inhibitor. (C) Western blot analysis of T47D cells treated at clonogenic density with IRES inhibitor cpd_W at $1 \mu \mathrm{g} / \mathrm{ml}$ as abovedescribed in (A). Whole cell lysates were collected as described in the legend of Fig. 8. Cx43, connexin 43 .

generate viable, cohesive colonies. Without the support of a cohesive colony, the majority of these cells eventually died, though tumor cell death under these conditions was insidious rather than acute. Notably, this concentration of cpd_W had no discernible adverse effects on the established populations of cells (Fig. 4).

Western blot analyses were performed on the low-density cells exposed to $1 \mu \mathrm{g} / \mathrm{ml} \mathrm{cpd} \_\mathrm{W}$, in an attempt to discern factors contributing to the failure of these cells to form viable colonies (Fig. 9C). The intensities of the $\alpha$-tubulin bands matched the quantitation of viable cells as displayed in the abovementioned time course graph. However, three notable variations from this pattern were observed. Firstly, induction of GRP78 and CHOP at a cpd_W concentration as low as $1 \mu \mathrm{g} / \mathrm{ml}$ served as an indication that sensitivity to IRES inhibition was heightened for cells at low density $(5-10 \mu \mathrm{g} / \mathrm{ml}$ cpd_W was required 


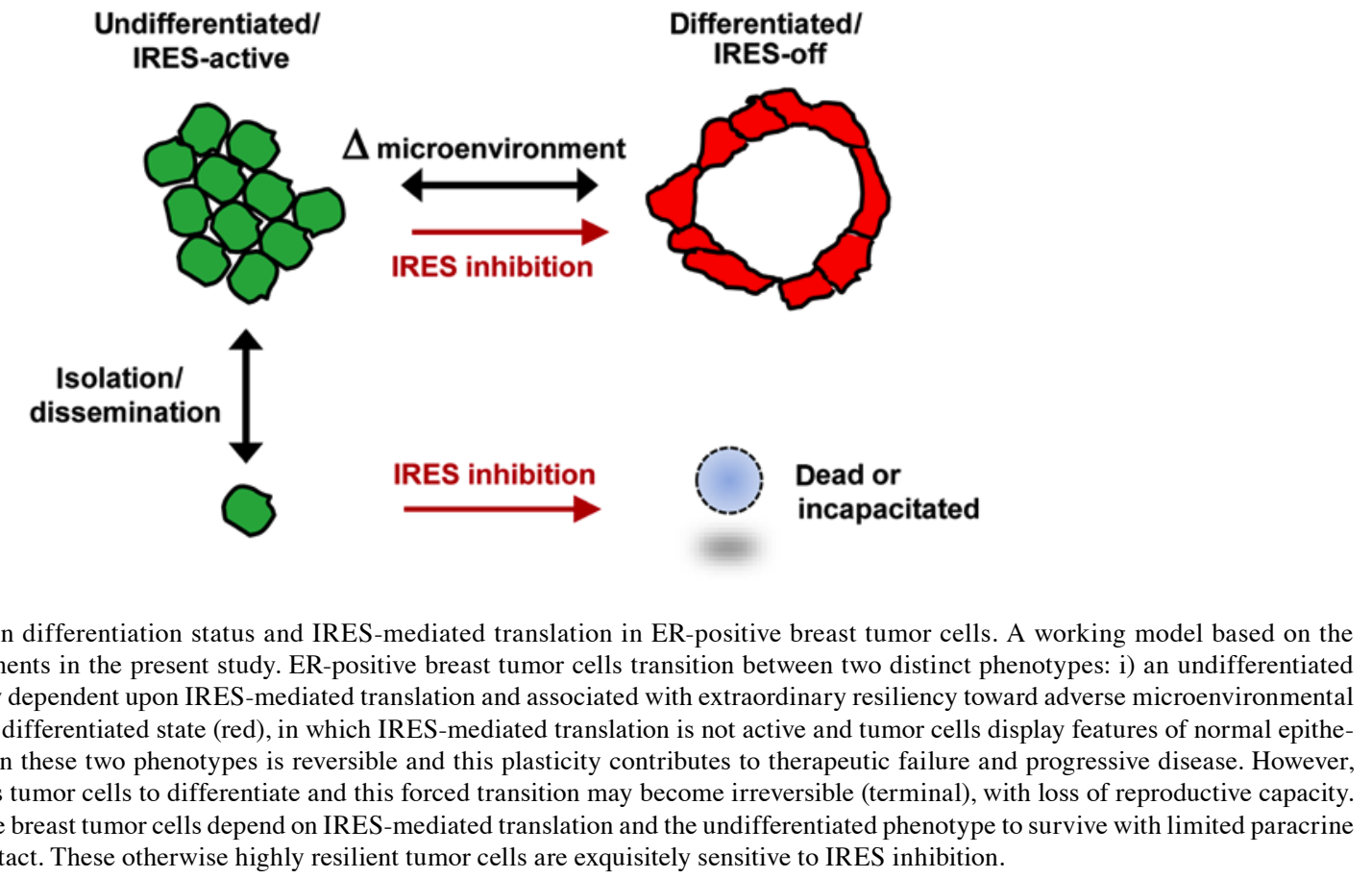

for the induction of these molecules in high-density cells). Secondly, connexin 43, which is known to be translated by an IRES and appeared in the western blot analyses of the highdensity tumor cell populations to be among the most sensitive proteins to the IRES inhibitor (Fig. 5), was completely undetectable at all time-points in the cpd_W-treated cells. Loss of the expression of connexin 43 and resulting impairment in gap junctional intercellular communication may have contributed significantly to failure of the treated cells to establish viable cohesive colonies. Thirdly, at the latest stage of the washout period, there was again evidence of a shift in Myc translation favoring synthesis of the p67 isoform, which has previously been associated with growth inhibition, differentiation and cell death.

\section{Discussion}

ER-positive breast tumor cells transition between two interconvertible phenotypic states distinguished by the degree of differentiation and use of IRES-mediated translation. A working model illustrating two distinct phenotypic states for ER-positive breast tumor cells, the transitions between them and the relationship to IRES-mediated translation is displayed in Fig. 10. Further investigation of these parameters in additional ER-positive breast tumor models and primary breast tumor specimens is warranted.

The results indicated that in ER-positive breast tumor cells, differentiation status is dynamic, subject to a reversible phenotypic transition between a moderately differentiated state and a relatively undifferentiated state. Imaging analyses allowed us to discern these variations in phenotype at the level of individual cells or groups of cells. The cells in the moderately differentiated state line acellular spaces, adopted specific spatial orientations with respect to adjacent cells and form highly contoured surfaces. Cells in the undifferentiated state exhibited no structural organization other than the propensity to cluster among themselves. The ER-positive breast tumor cells readily transitioned between these two distinct phenotypes, as a means of adapting to changes in microenvironment. The cells may be capable of shifting to the more resilient undifferentiated state when faced with challenging microenvironmental conditions such as limiting growth factors (e.g. serum deprivation). IRES-mediated translation is active and appears to be essential, for the undifferentiated state. Under more favorable conditions, a fraction of the tumor cells may adopt the differentiated state in which IRES-mediated translation is apparently not active. Chemical inhibition of IRES-mediated translation forced the cells to transition unilaterally to the differentiated IRES-off phenotype and this transition became irreversible. Thus, translational regulation and IRES-mediated translation in particular, may be an integral factor that distinguishes these phenotypic states.

Multiple biomarkers were capable of distinguishing these two distinct phenotypes. Each of the candidate biomarkers aligned precisely with one of these phenotypic states. The undifferentiated/IRES-active phenotype was IGF1R N20-positive and RACK1-negative and ER $\alpha$ was restricted to the cytoplasm. The differentiated/IRES-off phenotype was RACK1-positive and IGF1R N20-negative and ER $\alpha$, GRP78 and ZO-1 were all readily detected in the nucleus. These molecules and staining patterns may ultimately serve as diagnostic biomarkers applied to primary breast tumor specimens, to help gauge differentiation status and the degree to which an individual tumor or population of tumor cells relies on IRES-mediated translation. These candidate biomarkers could also potentially be used to investigate how IRES-mediated translation and differentiation status change in response to the adverse microenvironmental conditions experienced in vivo.

Of particular importance was the finding that cytoplasmic $\mathrm{ER} \alpha$ may serve as a biomarker for IRES-mediated translation in ER-positive breast tumor cells. Cytoplasmic ER $\alpha$ correlated with the undifferentiated phenotype in which the 
IRES-mediated translation was active, mirroring precisely the staining pattern observed with the antibody recognizing nascently translated IGF1R and inversely correlated with RACK1 epitope accessibility (as an indication of the IRES-off state). When cells transitioned to the differentiated phenotype, IRES-mediated translation was curtailed and ER $\alpha$ shifted entirely to the nucleus. ER $\alpha$ has two functional nuclear export sequences along with one nuclear localization signal, allowing it to shuttle between the nuclear and cytoplasmic compartments (23). Export of ER $\alpha$ to the cytoplasm is required for S-phase entry and cell proliferation (24) and blocking $\mathrm{ER} \alpha$ function decreases colony forming potential (25). Accumulation of ER $\alpha$ in the cytoplasm also correlates with resistance to hormonal therapy $(26,27)$. Characterization of non-nuclear (transcription-independent) functions of ER $\alpha$ is an active area of investigation, however, to our knowledge, a relationship between cytoplasmic ER $\alpha$ localization and IRESmediated translation has not been previously described. The functional significance of this relationship is not yet clear, however, it is of note that cytoplasmic ER $\alpha$ interacts with HSP90 (28), which appears to be involved in regulating IRESmediated translation (29). Additional research will be needed to determine whether ER $\alpha$ may actively promote the undifferentiated phenotype and/or IRES-mediated translation from its location in the cytoplasm.

The stark contrast in RACK1 epitope accessibility between the two phenotypic states indicated that RACK1 conformation and/or intermolecular interactions were distinctly different in the context of the clustered cells in which IRES-mediated translation was active and the differentiated cells in which IRES-mediated translation appeared to have been shut down. Cryo-EM images have placed RACK1 in close proximity to the mRNA exit site on the platform of the 40S subunit, where interactions with the 5'upstream regulatory region of the mRNA take place (13). Although RACK1 is an integral component of the $40 \mathrm{~S}$ ribosomal subunit, it is not required for general protein synthesis, but selectively promotes translation of specific mRNAs (several of which are known to be translated via an IRES, including MYC (30)). RACK1 is also required for the translation of certain viral IRESs (e.g. HCV) and RACK1 facilitates IRES-mediated translation in in vitro systems as well (14).

GRP78 and ZO-1 accompanied ER $\alpha$ in shifting from the cytoplasm to the nucleus upon cellular differentiation. GRP78 interacts with matrin-3 (a component of nuclear matrix) (31), although its function in the nucleus has not yet been thoroughly characterized. ZO-1 contains three nuclear localization signals and localizes to the nucleus of cells that lack full circumferential contact with other cells (32). ZO-1 interacts closely with the Y-box transcription factor ZONAB, which binds to the promoters of genes whose expression varies with cell density (33). ZO-1 has been observed in the nuclei of normal terminally differentiated epithelial cells at the tips of intestinal villi, post-mitotic cells which are preparing to undergo programmed cell death and exfoliation (32).

Immunofluorescence staining and confocal imaging demonstrated a marked increase in structural organization of E-cadherin and the microtubule cytoskeleton, consistent with the enhanced differentiation in the cells treated with the IRES inhibitor. Yet the western blot results indicated no net increase in the E-cadherin protein level. Thus, it appears that although some of the structural proteins needed for the transition to the terminally differentiated phenotype were already present in sufficient quantity, the cells may have been prevented from fully executing the differentiation program by one or more IRES-driven factors which perpetuated the undifferentiated state.

Notably, these two distinct phenotypes were at times observed even within an individual cell, where one region of the cytoplasm (bordering an open space, i.e. characteristic of a normal epithelial cell) revealed evidence of enhanced RACK1 epitope accessibility (indicative of the more differentiated/IRES-off state), while the cytoplasm on the other side of the cell (bordering a cluster of undifferentiated cells) showed evidence of active IRES-mediated translation (IGF1R N20 or cytoplasmic ER $\alpha$ immunoreactivity and RACK1 epitope masking). These biphenotypic cells were observed in vehicletreated wells (i.e. independent of IRES inhibition), therefore this subcellular phenotypic and translational specialization apparently has a physiological basis and is something these tumor cells readily employ. This pattern also suggests that, at least to some degree, phenotype is controlled at the cytoplasmic (i.e. translational) level.

Fundamental relationship between IRES-mediated translation and the undifferentiated state. We previously reported (12) that highly undifferentiated triple-negative breast tumor cells (as well as glioblastoma and osteosarcoma cells) were forced into terminal differentiation when continuously exposed to IRES inhibitor cpd_P for $>72 \mathrm{~h}$. The triple-negative breast tumor cells did not tolerate this transition to a fully differentiated phenotype and underwent a synchronized population-wide cell death event which was non-apoptotic and shared a number of features with cornification. In the ER-positive breast tumor cells, as little as $15-24 \mathrm{~h}$ exposure to cpd_W led to a terminal differentiation outcome which very closely resembled that observed in the triple-negative cells, with similar increases in polarity and structural organization. Unlike the triple-negative breast tumor cells however, the ER-positive breast tumor cells tolerated forced differentiation with only modest loss of viability, although their ability to transition back to the undifferentiated state and resume proliferation, which is of integral importance to their malignant behavior, was substantially compromised.

Thus, the impact of IRES inhibition on ER-positive and triple-negative breast tumor cells was actually quite similar. In both cases, the tumor cell population was forced to abandon the undifferentiated phenotype. The differences in timing of response and final outcome reflected the inherent differences in the phenotypic starting point for these cells. ER-positive breast tumor cells are intrinsically capable of transitioning reversibly to a differentiated state and may not require as great a stimulus to initiate terminal differentiation, while the triplenegative breast tumor cells are phenotypically further removed from the terminally differentiated state and therefore required prolonged deprivation of IRES-mediated translation in order to revoke the undifferentiated phenotype. Collectively, these results provided further evidence that IRES-mediated translation is critical for the maintenance of the undifferentiated state. 
Clinical implications and potential therapeutic applications of IRES inhibition in ER-positive breast cancer. IRES inhibition exerted profound, detrimental effects on ER-positive breast tumor cells, inducing terminal differentiation in highdensity tumor cell populations and complete loss of clonogenic survival (either massive cell death or incapacitation) in isolated or low-density tumor cells. In all cases, the tumor cells lost the ability to perpetuate the malignant clone. Thus, each of these could represent a clinically beneficial outcome, illustrating the potential use of IRES inhibition as a therapeutic strategy.

Low-density tumor cells were particularly vulnerable to IRES inhibition because they rely on the undifferentiated phenotype and IRES-mediated translation to survive when paracrine support and intercellular contact were limited. The stringent experimental protocols used in the present study were intended to model the adverse microenvironmental conditions encountered by disseminated (i.e. micrometastatic) tumor cells in vivo. The fact that the ER-positive breast tumor cells were able to survive and form colonies at low cell density concomitant with acute serum deprivation was indicative of the extraordinary resiliency of these cells. Although frequently tagged as 'less aggressive', we found that the isolated ER-positive breast tumor cells are actually more resilient than even triple-negative breast tumor cells, which do not survive such conditions.

This resiliency is reflected in the natural history of ER-positive breast cancer, where a substantial proportion of patients fail to achieve long term remission with post-surgical hormonal therapy and instead experience repeated recurrences and progressive metastatic disease $(34,35)$. The potent inhibition of clonogenic survival by very low concentrations of cpd_W suggests that IRES inhibition may be used to effectively incapacitate or eliminate these otherwise highly resilient ER-positive breast tumor cells, thereby filling a critical gap in the therapeutic armamentarium.

\section{Acknowledgements}

Not applicable.

\section{Funding}

The present study was supported by the National Institutes of Health (grant no. R01CA108886), the American Society for Clinical Oncology Young Investigator Award (to CV), Susan G Komen Career Catalyst Award in Basic and Translational Research (grant no. CCR15331062 to CV), the UAB Comprehensive Cancer Center Drug Discovery and Development Program and the Alabama Drug Discovery Alliance.

\section{Availability of data and materials}

The analyzed datasets generated during the study are available from the corresponding author on reasonable request.

\section{Authors' contributions}

$\mathrm{CV}$ and SWB performed the experiments and wrote the manuscript. $\mathrm{ZM}$ and $\mathrm{HC}$ made substantial contributions to conception, design and intellectual content of the studies. KRZ, SLS, and WEG made key contributions to analysis and interpretation of data. All authors read and approved the final manuscript.

\section{Ethics approval and consent to participate}

This study was approved by the UAB Institutional Review Board (project no. X040506011).

\section{Consent for publication}

Not applicable.

\section{Competing interests}

The authors declare that they have no competing interests.

\section{References}

1. Prats AC and Prats H: Translational control of gene expression: Role of IRESs and consequences for cell transformation and angiogenesis. Prog Nucleic Acid Res Mol Biol 72: 367-413, 2002.

2. Baird SD, Turcotte M, Korneluk RG and Holcik M: Searching for IRES. RNA 12: 1755-1785, 2006.

3. Spriggs KA, Stoneley $M$, Bushell $M$ and Willis AE Re-programming of translation following cell stress allows IRESmediated translation to predominate. Biol Cell 100: 27-38, 2008.

4. Meng Z, Jackson NL, Choi H, King PH, Emanuel PD and Blume SW: Alterations in RNA-binding activities of IRESregulatory proteins as a mechanism for physiological variability and pathological dysregulation of IGF-IR translational control in human breast tumor cells. J Cell Physiol 217: 172-183, 2008.

5. Nagamachi A, Htun PW, Ma F, Miyazaki K, Yamasaki N, Kanno M, Inaba T, Honda Z, Okuda T, Oda H, et al: A 5'untranslated region containing the IRES element in the Runx1 gene is required for angiogenesis, hematopoiesis and leukemogenesis in a knock-in mouse model. Dev Biol 345: 226-236, 2010.

6. Blau L, Knirsh R, Ben-Dror I, Oren S, Kuphal S, Hau P, Proescholdt M, Bosserhoff AK and Vardimon L: Aberrant expression of c-Jun in glioblastoma by internal ribosome entry site (IRES)-mediated translational activation. Proc Natl Acad Sci USA 109: E2875-E2884, 2012.

7. Dobson T, ChenJ and KrushelLA: Dysregulating IRES-dependent translation contributes to over-expression of the Aurora A kinase onco-protein. Mol Cancer Res 11: 887-900, 2013.

8. Lynch SR and Puglisi JD: Structural origins of aminoglycoside specificity for prokaryotic ribosomes. J Mol Biol 306: 1037-1058, 2001.

9. Hansen JL, Moore PB and Steitz TA: Structures of five antibiotics bound to the peptidyl transferase center of the large ribosomal subunit. J Mol Biol 330: 1061-1075, 2003.

10. Baselga J, Campone M, Piccart M, Burris HA III, Rugo HS, Sahmoud T, Noguchi S, Gnant M, Pritchard KI, Lebrun F, et al: Everolimus in postmenopausal hormone-receptor-positive advanced breast cancer. N Engl J Med 366: 520-529, 2012.

11. Vaklavas C, Meng Z, Choi H, Grizzle WE, Zinn KR and Blume SW: Small molecule inhibitors of IRES-mediated translation. Cancer Biol Ther 16: 1471-1485, 2015.

12. Vaklavas C, Grizzle WE, Choi H, Meng Z, Zinn KR, Shrestha K and Blume SW: IRES inhibition induces terminal differentiation and synchronized death in triple-negative breast cancer and glioblastoma cells. Tumour Biol 37: 13247-13264, 2016.

13. Sengupta J, Nilsson J, Gursky R, Spahn CM, Nissen P and Frank J: Identification of the versatile scaffold protein RACK1 on the eukaryotic ribosome by cryo-EM. Nat Struct Mol Biol 11: 957-962, 2004.

14. Majzoub K, Hafirassou ML, Meignin C, Goto A, Marzi S, Fedorova A, Verdier Y, Vinh J, Hoffmann JA, Martin F, et al: RACK1 controls IRES-mediated translation of viruses. Cell 159: 1086-1095, 2014

15. Barraille P, Chinestra P, Bayard F and Faye JC: Alternative initiation of translation accounts for a $67 / 45 \mathrm{kDa}$ dimorphism of the human estrogen receptor ERalpha. Biochem Biophys Res Commun 257: 84-88, 1999. 
16. Vedrenne C, Klopfenstein DR and Hauri HP: Phosphorylation controls CLIMP-63-mediated anchoring of the endoplasmic reticulum to microtubules. Mol Biol Cell 16: 1928-1937, 2005.

17. Nikonov AV, Hauri HP, Lauring B and Kreibich G: Climp63-mediated binding of microtubules to the ER affects the lateral mobility of translocon complexes. J Cell Sci 120: 2248-2258, 2007.

18. Schiavi A, Hudder A and Werner R: Connexin 43 mRNA contains a functional internal ribosome entry site. FEBS Lett 464 118-122, 1999.

19. Marash L, Liberman N, Henis-Korenblit S, Sivan G, Reem E, Elroy-Stein $O$ and Kimchi A: DAP5 promotes cap-independent translation of Bcl-2 and CDK1 to facilitate cell survival during mitosis. Mol Cell 30: 447-459, 2008.

20. Spandidos DA, Pintzas A, Kakkanas A, Yiagnisis M, Mahera H, Patra E and Agnantis NJ: Elevated expression of the myc gene in human benign and malignant breast lesions compared to normal tissue. Anticancer Res 7: 1299-1304, 1987.

21. Hann SR, Dixit M, Sears RC and Sealy L: The alternatively initiated c-Myc proteins differentially regulate transcription through a noncanonical DNA-binding site. Genes Dev 8: 2441-2452, 1994.

22. Benassayag C, Montero L, Colombié N, Gallant P, Cribbs D and Morello D: Human c-Myc isoforms differentially regulate cell growth and apoptosis in Drosophila melanogaster. Mol Cell Biol 25: 9897-9909, 2005.

23. Tecalco-Cruz AC, Pérez-Alvarado IA, Ramírez-Jarquín JO and Rocha-Zavaleta L: Nucleo-cytoplasmic transport of estrogen receptor alpha in breast cancer cells. Cell Signal 34: 121-132, 2017.

24. Lombardi M, Castoria G, Migliaccio A, Barone MV, Di Stasio R, Ciociola A, Bottero D, Yamaguchi H, Appella E and Auricchio F: Hormone-dependent nuclear export of estradiol receptor and DNA synthesis in breast cancer cells. J Cell Biol 182: 327-340, 2008.

25. Basak P, Chatterjee S, Weger S, Bruce MC, Murphy LC and Raouf A: Estrogen regulates luminal progenitor cell differentiation through $\mathrm{H} 19$ gene expression. Endocr Relat Cancer 22 : 505-517, 2015

26. Fan P, Wang J, Santen RJ and Yue W: Long-term treatment with tamoxifen facilitates translocation of estrogen receptor alpha out of the nucleus and enhances its interaction with EGFR in MCF-7 breast cancer cells. Cancer Res 67: 1352-1360, 2007.
27. Guest SK, Ribas R, Pancholi S, Nikitorowicz-Buniak J, Simigdala N, Dowsett M, Johnston SR and Martin LA: Src is a potential therapeutic target in endocrine-resistant breast cancer exhibiting low estrogen Receptor-mediated transactivation. PLoS One 11: e0157397, 2016.

28. Dhamad AE, Zhou Z, Zhou J and Du Y: Systematic proteomic identification of the heat shock proteins (Hsp) that interact with estrogen receptor alpha $(\mathrm{ER} \alpha)$ and biochemical characterization of the ER $\alpha$-Hsp70 interaction. PLoS One 11: e0160312, 2016.

29. Yang HW, Kim TM, Song SS, Menon L, Jiang X, Huang W, Black PM, Park PJ, Carroll RS and Johnson MD: A small subunit processome protein promotes cancer by altering translation. Oncogene 34: 4471-4481, 2015.

30. Ruan Y, Sun L, Hao Y, Wang L, Xu J, Zhang W, Xie J, Guo L, Zhou L, Yun X, et al: Ribosomal RACK1 promotes chemoresistance and growth in human hepatocellular carcinoma. J Clin Invest 122: 2554-2566, 2012.

31. Osman AM and van Loveren H: Matrin 3 co-immunoprecipitates with the heat shock proteins glucose-regulated protein 78 (GRP78), GRP75 and glutathione S-transferase $\pi$ isoform 2 (GST 2 2) in thymoma cells. Biochimie 101: 208-214, 2014.

32. Gottardi CJ, Arpin M, Fanning AS and Louvard D: The junctionassociated protein, zonula occludens-1, localizes to the nucleus before the maturation and during the remodeling of cell-cell contacts. Proc Natl Acad Sci USA 93: 10779-10784, 1996.

33. Balda MS, Garrett MD and Matter K: The ZO-1-associated Y-box factor ZONAB regulates epithelial cell proliferation and cell density. J Cell Biol 160: 423-432, 2003.

34. Clarke R, Tyson JJ and Dixon JM: Endocrine resistance in breast cancer-An overview and update. Mol Cell Endocrinol 418: 220-234, 2015.

35. Murphy CG and Dickler MN: Endocrine resistance in hormoneresponsive breast cancer: Mechanisms and therapeutic strategies. Endocr Relat Cancer 23: R337-R352, 2016.

This work is licensed under a Creative Commons Attribution-NonCommercial-NoDerivatives 4.0 International (CC BY-NC-ND 4.0) License. 